\title{
4. MINERAL AND CHEMICAL COMPOSITION OF SEDIMENTS OF THE VORING PLATEAU, DSDP LEG 38
}

\author{
E.M. Emelyanov, A.I. Blazchishin, G.S. Kharin, N.G. Lozovaya, and K.P: Zangalis, \\ Atlantic Branch, P.P. Shirshov Institute of Oceanology, Academy of Sciences of the USSR, Kaliningrad
}

\section{INTRODUCTION}

Samples from five sites, located in the northwest part of the V $\phi$ ring Plateau (Figure 1), were investigated. The studies included:

1) grain size composition of six fractions $(>0.1 ; 0.1-$ $0.05 ; 0.05-0.01 ; 0.01-0.05 ; 0.005-0.001$, and $<0.001$ $\mathrm{mm}$ );

2) mineral composition of light and heavy minerals (S.G. $>2.9$ of fractions $0.1-0.05 \mathrm{~mm}$ );

3) determination of calcite, aragonite, dolomite, siderite, quartz, plagioclase, and potassic feldspars; and

4) chemical composition and the content of $\mathrm{CaCO}_{3}$, $\mathrm{C}_{\text {org }}, \mathrm{Fe}, \mathrm{Mn}, \mathrm{Ti}, \mathrm{P}, \mathrm{Cu}, \mathrm{Zn}, \mathrm{Ni}, \mathrm{Co}, \mathrm{Cr}, \mathrm{Cd}$ (by spectrophotometer), $\mathrm{K}_{2} \mathrm{O}$ and $\mathrm{Na}_{2} \mathrm{O}$ (by plasmatic photometer M-3).

In this paper, and accompanying figures and tables, the sediment terminology is that of the authors and may not correspond to shipboard designations. Sample numbers are those assigned by the authors for the investigations.

\section{GRAIN SIZE}

According to the preliminary core descriptions (ICD) and more exact descriptions by other investigators in shore-based laboratories, sediments of the V申ring Plateau are represented mainly by marine clays and silty clay muds; only in exceptional cases by sands and silts. This is confirmed by the grain-size analyses (Table 1).

The largest number of sandy interbeds and the $>0.1$ mm fraction is present in "glacial" deposits at Site 343. This may be a result of the accumulation of sediments, not only by glacial-marine processes, but by density currents moving downslope to the base of the V $\phi$ ring Plateau. The clay fraction is abundant in Plio/Pleistocene and Eocene and Oligocene deposits of Sites 339 and 340 . This is due to the location of the sites between the V $\phi$ ring Plateau and Scandinavia, where quiet sedimentation conditions were present.

Genetically, on the basis of grain size, there are six sediment varieties: (1) glacial marine; (2) terrigenous, slightly calcareous terrigenous, and slightly calcareous with a $\mathrm{CaCO}_{3}$ or $\mathrm{SiO}_{2}$ (amorph) content equal to $10 \%$ $30 \%$; (3) diatomaceous ( $>30 \% \quad \mathrm{SiO}_{2}$ [amorph]); (4) mixed siliceous/diatomaceous-spicules, spiculesradiolarian ( $>30 \% \mathrm{SiO}_{2}$ [amorph]); (5) diatomites; and

(6) calcareous-foraminiferal $\left(>50 \% \mathrm{CaCO}_{3}\right)$.

\section{MINERAL COMPOSITION OF THE 0.1-0.05 MM FRACTION}

The amount of the heavy subfraction is usually small, however, in some horizons it increases to $20 \%-30 \%$.
This is not a result of volcanic minerals, as it is normal, but is due to the presence of authigenic sulfides. Terrigenous minerals, which are abundant in the heavy subfractions, are represented mainly by ore minerals: magnetite, titanomagnetite, ilmenite, plus pyroxenes, hornblende, epidote, garnet, and rutile (Table 2, Figure 2).

Magnetite is present as angular to round formless grains, with a metallic luster, and well-developed magnetic properties. Ilmenite usually is strongly leucoxenizated, but has a table-like form. In contrast to magnetite, titanomagnetite is oxidized and has no magnetic properties.

Clinopyroxenes are colorless to light brown short prisms, with indented rims. Sometimes orthopyroxene (hypersthene) is present with a characteristic light green to light pink pleochroism. Hornblende is represented by green and brown varieties; alkaline varieties are rare. Clinozoisite represents the epidote group. Usually it forms angular, anhedral grains, pistachio-colored, with anomalous interference colors. Garnet is present as angular, isotropic grains with a high relief, colorless or pink. Anomalous garnet is present.

Terrigenous minerals of light fraction are mainly represented by quartz, feldspar, mica, and clay (minerals?) particles. The feldspars have various compositions; plagioclases (sodic), as well as potassic feldspar. The feldspar grains are usually rounded and clay minerals are mainly formed from feldspar alteration. Brown biotite and colorless muscovite are the abundant micas. Greenish chlorite is present as single grains.

Volcanic minerals are mainly represented by colorless volcanic glass and are acidic $(N=1.502$ to 1.515$)$. This is equal to an $\mathrm{SiO}_{2}$ content of $64 \%-71 \%$. Greenishbrown and brown glass is rare. The glass is present as sharp, angular fragments with a conchoidal fracture, and contains vesicles with ferrous sulfides. Volcanic ash is represented by cloudy, black, brown, formless grains.

Authigenic minerals consist of carbonates, glauconite, ferrous sulfides, and barite. There are two carbonate varieties: calcite and siderite. The brown ferruginous variety is restricted to the heavy fraction, calcite to the light. Their authigenic origin is confirmed by the grain form, which consists of rose-like crystals with the noticeable growth center. Glauconite is present as oval, green grains with a specific aggregate polarization. Fe-sulfides are abundant in some horizons, and comprise $20 \%-30 \%$ of the heavy fraction. They develop upon volcanic glass or ash fragments, or in diatom shells.

Figure 2 shows the content of biogenic carbonates and siliceous remains. The carbonates are mainly 


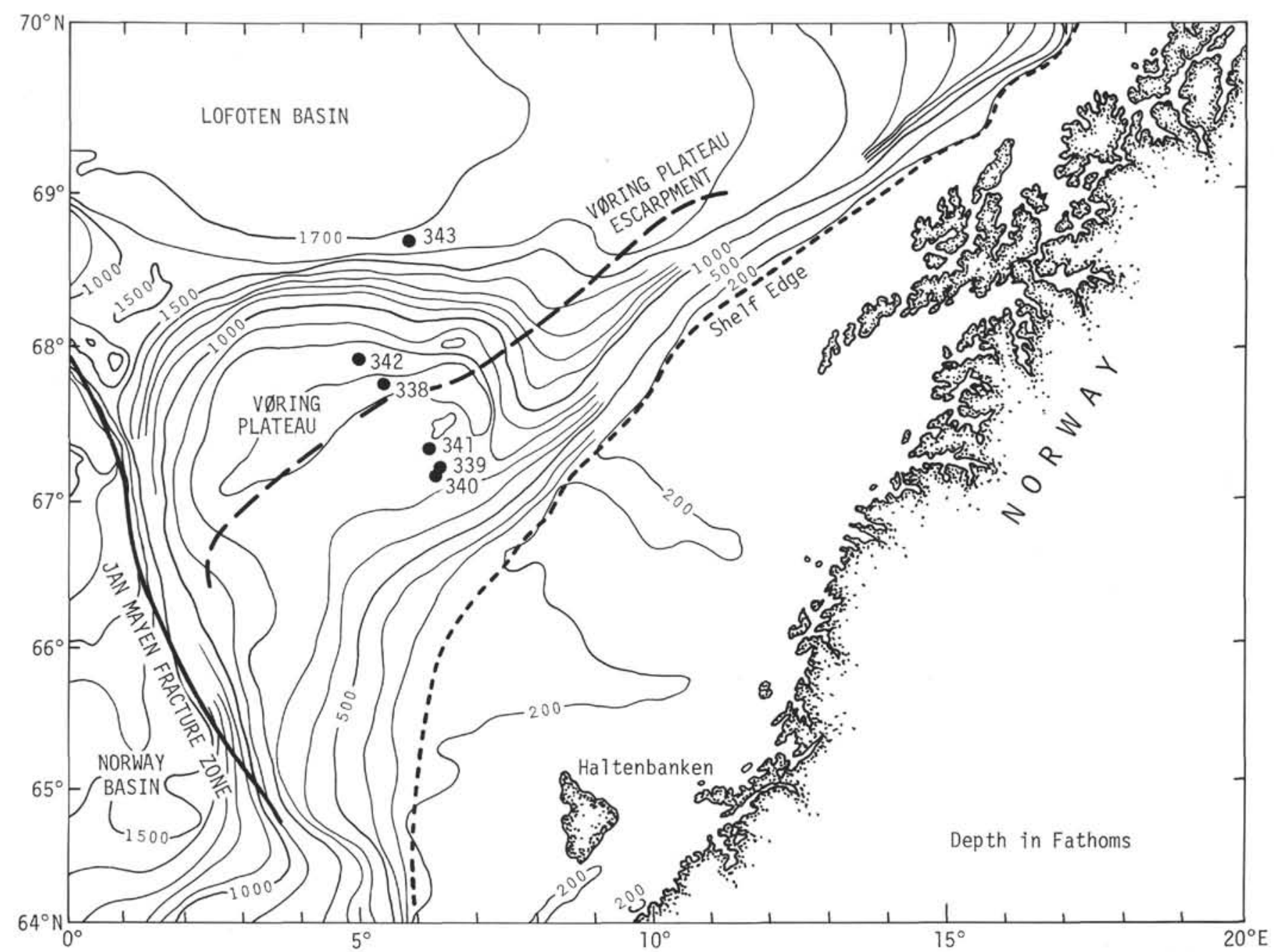

Figure 1. Location of DSDP Leg 38 sites drilled on the Vhring Plateau.

represented by fragments of foraminifera shells, the siliceous remains by fragments of diatoms, Radiolaria, and sponges.

\section{DISTRIBUTION OF MINERALS IN COLUMN OF SEDIMENTARY STRATA}

In Pleistocene/Pliocene deposits (Table 3, Figure 2) there is an increased content of hornblende, clinopyroxene, augite, epidote, garnet, quartz, feldspar, tourmaline, staurolite, and rutile. These represent minerals of mixed origin; sedimentary, metamorphic, and magmatic. This is expected because all Scandinavian continental rocks were subjected to glacial erosion. Only two glauconite peaks are noted (Sites 339 and 343, Figure 2) and are probably a result of diagenetic processes as indicated by the increased contents of foraminifera (Site 343), diatoms, Radiolaria, spicules (Site 339).

There is an increase in the content of volcanic glass, Fe-sulfides, clayey aggregates which define Pliocene, Miocene, and Oligocene deposits, cored at Sites 341 and 342. The contents of ilmenite, pyroxenes, hornblende, epidote, garnet, quartz, feldspar are sharply increased in comparison with the overlying Plio-Pleisto- cene sediments. These data may indicate that, in the formation of Miocene sediments on the V $\phi$ ring Plateau, volcanism was more dominant than in the Plio-Pleistocene. An increased content of authigenic minerals is also characteristic of the Miocene. Besides the Fesulfides (Site 343, 147.5 m), an increase of glauconite (up to $11 \%$ ) and biogenic siliceous particles is noted.

Generally, the regularities of mineral distribution in the Eocene are the same for the Neogene, i.e., the content of volcanic minerals, authigenic Fe-sulfides increase, and the amount of terrigenous minerals, especially of the heavy fraction, is lower. However, in some early Eocene horizons (Site 343 ) a high feldspar and ilmenite content is observed. This is indicative of an increased influx of terrigenous sediments during some phases of Eocene sedimentation.

\section{BULK MINERAL DISTRIBUTION (X-RAY)}

\section{Carbonates}

The carbonates generally are characteristic for Pleistocene sediments. Calcite is abundant, with other calcareous minerals found in trace concentrations in a small number of samples. However, at Site 342 (2-1) on 
TABLE 1

Grain Size (\%) of Sediments from the V $\phi$ ring Plateau, DSDP Leg 38

\begin{tabular}{|c|c|c|c|c|c|c|c|c|c|}
\hline \multirow{2}{*}{$\begin{array}{l}\text { Sample } \\
\text { (Interval } \\
\text { in } \mathrm{cm} \text { ) }\end{array}$} & \multirow[b]{2}{*}{$\begin{array}{c}\text { Deptha } \\
\text { (m) }\end{array}$} & \multirow{2}{*}{$\begin{array}{l}\text { Grain-Size } \\
\text { Classification of } \\
\text { Sediments }\end{array}$} & \multicolumn{7}{|c|}{ Fractions, mm (\%) } \\
\hline & & & $>0.1$ & $\begin{array}{l}0.1- \\
0.05\end{array}$ & $\begin{array}{c}-0.05- \\
0.01\end{array}$ & $\begin{array}{l}0.01- \\
0.005\end{array}$ & $\begin{array}{c}0.005- \\
0.001\end{array}$ & $<0.001$ & $<0.01$ \\
\hline \multicolumn{10}{|l|}{ Site 339} \\
\hline $\begin{array}{l}2-1,119-121 \\
2-3,129-131 \\
3-4,142-144 \\
4-2,121-123 \\
5-1,139-140 \\
6-3,102-104 \\
7-1,130-132 \\
8-1,100-102 \\
10-3,70-73 \\
12-2,70-72\end{array}$ & $\begin{array}{r}9.2 \\
12.4 \\
23.7 \\
33.0 \\
38.0 \\
49.9 \\
56.9 \\
66.0 \\
87.9 \\
104.2\end{array}$ & $\begin{array}{l}\text { Pelitic mud } \\
\text { Pelitic mud } \\
\text { Pelitic mud } \\
\text { Pelitic mud } \\
\text { Aleuritic-pelitic mud } \\
\text { Aleuritic-pelitic mud } \\
\text { Aleuritic-pelitic mud } \\
\text { Aleuritic-pelitic mud } \\
\text { Pelitic mud } \\
\text { Pelitic mud }\end{array}$ & $\begin{array}{l}2.8 \\
3.1 \\
7.8 \\
6.9 \\
9.6 \\
6.6 \\
7.4 \\
7.3 \\
0.8 \\
0.3\end{array}$ & $\begin{array}{r}2.7 \\
2.2 \\
6.6 \\
5.4 \\
13.1 \\
11.9 \\
10.4 \\
8.1 \\
2.5 \\
0.3\end{array}$ & $\begin{array}{r}10.6 \\
9.6 \\
13.2 \\
11.9 \\
20.9 \\
24.6 \\
20.1 \\
15.5 \\
6.0 \\
3.0\end{array}$ & $\begin{array}{l}12.0 \\
24.3 \\
19.8 \\
19.8 \\
21.1 \\
18.2 \\
31.1 \\
29.6 \\
11.2 \\
12.2\end{array}$ & $\begin{array}{l}32.0 \\
24.4 \\
23.0 \\
33.0 \\
21.1 \\
22.7 \\
31.1 \\
22.2 \\
56.8 \\
36.2\end{array}$ & $\begin{array}{r}39.9 \\
36.5 \\
29.6 \\
23.0 \\
14.1 \\
15.9 \\
0.0 \\
17.3 \\
22.7 \\
48.1\end{array}$ & $\begin{array}{l}83.9 \\
85.2 \\
72.4 \\
75.8 \\
56.3 \\
56.8 \\
62.2 \\
69.1 \\
90.7 \\
96.5\end{array}$ \\
\hline \multicolumn{10}{|l|}{ Site 340} \\
\hline $\begin{array}{l}1, \mathrm{CC} \\
2-1,38-40 \\
2-3,75-77 \\
3-3,75-77 \\
4-4,75-77 \\
5-2,70-72 \\
6-3,60-62 \\
7-1,95-97 \\
7-6,95-97 \\
8-4,72-74 \\
9-3,70-72 \\
10-2,50-52 \\
11-4,81-83\end{array}$ & $\begin{array}{r}9.5 \\
9.9 \\
13.5 \\
23.0 \\
34.0 \\
40.3 \\
51.3 \\
58.0 \\
65.0 \\
72.0 \\
79.9 \\
87.6 \\
100.7\end{array}$ & $\begin{array}{l}\text { Pelitic mud } \\
\text { Pelitic mud } \\
\text { Pelitic mud } \\
\text { Pelitic mud } \\
\text { Pelitic mud } \\
\text { Pelitic mud } \\
\text { Pelitic mud } \\
\text { Pelitic mud } \\
\text { Pelitic mud } \\
\text { Pelitic mud } \\
\text { Pelitic mud } \\
\text { Pelitic mud } \\
\text { Pelitic mud }\end{array}$ & $\begin{array}{l}4.2 \\
4.1 \\
0.6 \\
0.8 \\
0.4 \\
7.4 \\
0.5 \\
0.6 \\
0.5 \\
4.2 \\
1.4 \\
2.1 \\
5.1\end{array}$ & $\begin{array}{l}4.4 \\
4.5 \\
6.1 \\
0.5 \\
1.3 \\
4.5 \\
1.3 \\
2.4 \\
3.1 \\
2.0 \\
2.8 \\
5.6 \\
1.8\end{array}$ & $\begin{array}{r}9.9 \\
14.5 \\
11.6 \\
6.3 \\
14.7 \\
13.5 \\
7.0 \\
8.8 \\
5.4 \\
10.1 \\
14.1 \\
15.4 \\
16.8\end{array}$ & $\begin{array}{l}17.9 \\
32.9 \\
27.2 \\
34.6 \\
41.5 \\
44.9 \\
60.8 \\
35.1 \\
33.9 \\
37.2 \\
20.5 \\
38.5 \\
17.0\end{array}$ & $\begin{array}{l}31.8 \\
33.0 \\
13.6 \\
34.8 \\
25.5 \\
29.8 \\
15.2 \\
35.4 \\
34.2 \\
28.0 \\
40.8 \\
19.1 \\
34.0\end{array}$ & $\begin{array}{r}31.8 \\
11.0 \\
40.9 \\
23.0 \\
16.6 \\
0.0 \\
15.2 \\
17.7 \\
22.8 \\
18.5 \\
20.5 \\
19.4 \\
25.3\end{array}$ & $\begin{array}{l}81.5 \\
76.9 \\
81.7 \\
92.4 \\
83.6 \\
74.7 \\
91.2 \\
88.2 \\
90.9 \\
83.7 \\
81.8 \\
77.0 \\
76.3\end{array}$ \\
\hline \multicolumn{10}{|l|}{ Site 341} \\
\hline $\begin{array}{l}1-3,75-77 \\
2-2,75-77 \\
4-1,75-77 \\
6-3,50 \\
7-3,50 \\
8-3,50 \\
10-1,109-111 \\
13-1,142-144 \\
17-2,129-131 \\
20-2,100-102 \\
21-4,90-92 \\
28-3,100-102 \\
29-4,118-120 \\
30-4,41-43 \\
32-4,50-52\end{array}$ & $\begin{array}{l}3.4 \\
11.9 \\
29.3 \\
51.25 \\
60.8 \\
70.3 \\
86.7 \\
107.0 \\
183.5 \\
240.2 \\
262.3 \\
393.9 \\
405.1 \\
413.7 \\
432.9\end{array}$ & $\begin{array}{l}\text { Pelitic mud } \\
\text { Pelitic mud } \\
\text { Pelitic mud } \\
\text { Fine-aleuritic mud } \\
\text { Pelitic mud } \\
\text { Aleuritic-pelitic mud } \\
\text { Fine-aleuritic mud } \\
\text { Aleuritic-pelitic mud } \\
\text { Fine-aleuritic mud } \\
\text { Pelitic mud } \\
\text { Pelitic mud } \\
\text { Aleuritic-pelitic mud } \\
\text { Aleuritic-pelitic mud } \\
\text { Aleuritic-pelitic mud } \\
\text { Aleuritic-pelitic mud }\end{array}$ & $\begin{array}{r}2.1 \\
9.0 \\
5.2 \\
0.8 \\
2.8 \\
15.0 \\
11.2 \\
9.3 \\
16.2 \\
2.2 \\
2.1 \\
0.6 \\
0.2 \\
0.6 \\
0.4\end{array}$ & $\begin{array}{r}4.9 \\
4.2 \\
4.5 \\
5.0 \\
4.5 \\
13.5 \\
9.6 \\
7.8 \\
10.7 \\
2.0 \\
3.6 \\
1.0 \\
0.9 \\
1.1 \\
0.6\end{array}$ & $\begin{array}{l}17.5 \\
14.8 \\
11.7 \\
68.7 \\
14.8 \\
26.5 \\
34.9 \\
23.7 \\
33.7 \\
21.6 \\
16.6 \\
37.6 \\
32.2 \\
42.9 \\
29.7\end{array}$ & $\begin{array}{r}25.2 \\
21.0 \\
19.7 \\
6.4 \\
15.6 \\
7.0 \\
11.6 \\
8.9 \\
6.1 \\
14.4 \\
8.6 \\
17.9 \\
15.9 \\
18.5 \\
15.1\end{array}$ & $\begin{array}{r}32.4 \\
33.0 \\
34.4 \\
9.6 \\
46.7 \\
19.0 \\
18.7 \\
22.2 \\
21.2 \\
33.5 \\
41.0 \\
28.6 \\
25.4 \\
14.8 \\
21.1\end{array}$ & $\begin{array}{r}18.0 \\
18.0 \\
24.6 \\
9.5 \\
15.6 \\
19.0 \\
13.9 \\
28.1 \\
12.1 \\
26.4 \\
28.1 \\
14.2 \\
25.4 \\
22.1 \\
33.1\end{array}$ & $\begin{array}{l}75.6 \\
72.0 \\
78.7 \\
25.5 \\
77.9 \\
45.0 \\
44.2 \\
59.2 \\
39.4 \\
74.3 \\
77.7 \\
60.7 \\
66.7 \\
55.4 \\
69.3\end{array}$ \\
\hline \multicolumn{10}{|l|}{ Site 342} \\
\hline $\begin{array}{l}1-2,49-51 \\
1-5,69-71 \\
2-2,94-96 \\
3-4,100-102 \\
4-2,110-112 \\
5-2,100-102 \\
5-6,100-102 \\
6-4,61-62\end{array}$ & $\begin{array}{r}2.0 \\
6.7 \\
40.1 \\
90.9 \\
125.8 \\
135.2 \\
141.5 \\
147.5\end{array}$ & $\begin{array}{l}\text { Aleuritic-pelitic mud } \\
\text { Aleuritic-pelitic mud } \\
\text { Aleuritic-pelitic mud } \\
\text { Fine-aleuritic mud } \\
\text { Aleuritic-pelitic mud } \\
\text { Aleuritic-pelitic mud } \\
\text { Aleuritic-pelitic mud } \\
\text { F-ine-aleuritic mud }\end{array}$ & $\begin{array}{r}10.2 \\
7.2 \\
24.5 \\
2.9 \\
1.4 \\
1.0 \\
1.0 \\
21.7\end{array}$ & $\begin{array}{r}10.3 \\
7.3 \\
4.8 \\
8.1 \\
6.0 \\
4.9 \\
5.7 \\
5.5\end{array}$ & $\begin{array}{l}20.7 \\
16.9 \\
15.0 \\
41.8 \\
37.6 \\
28.1 \\
35.4 \\
34.1\end{array}$ & $\begin{array}{r}8.4 \\
8.6 \\
11.1 \\
7.8 \\
11.8 \\
16.6 \\
11.6 \\
16.1\end{array}$ & $\begin{array}{r}37.8 \\
37.2 \\
29.0 \\
27.6 \\
23.6 \\
22.0 \\
23.2 \\
9.6\end{array}$ & $\begin{array}{l}12.6 \\
22.9 \\
15.6 \\
11.8 \\
19.6 \\
27.5 \\
23.0 \\
12.9\end{array}$ & $\begin{array}{l}58.8 \\
68.7 \\
55.7 \\
47.2 \\
55.0 \\
66.1 \\
57.8 \\
38.6\end{array}$ \\
\hline \multicolumn{10}{|l|}{ Site 343} \\
\hline $\begin{array}{l}1-1,75-77 \\
1-3,75-77 \\
2-2,105-107 \\
2, C C \\
2-4,75-77 \\
3-2,129-131 \\
3-4,129-131 \\
3-6,119-121 \\
4-3,110-112 \\
5-2,19-21 \\
7-0,30-32 \\
8-3,100-103 \\
11-2,130-132 \\
15-1,99-101 \\
16-3,18-20\end{array}$ & $\begin{array}{r}0.25 \\
1.25 \\
5.7 \\
12.5 \\
8.7 \\
53.5 \\
56.0 \\
59.7 \\
102.6 \\
147.3 \\
202.0 \\
216.3 \\
243.5 \\
270.1 \\
282.0\end{array}$ & $\begin{array}{l}\text { Aleuritic-pelitic mud } \\
\text { Aleuritic-pelitic mud } \\
\text { Aleuritic-pelitic mud } \\
\text { Aleuritic-pelitic mud } \\
\text { Aleuritic-pelitic mud } \\
\text { Sand } \\
\text { Fine-aleuritic mud } \\
\text { Sand } \\
\text { Aleuritic-pelitic mud } \\
\text { Aleuritic-pelitic mud } \\
\text { Aleuritic-pelitic mud } \\
\text { Aleuritic-pelitic mud } \\
\text { Aleuritic-pelitic mud } \\
\text { Aleuritic-pelitic mud } \\
\text { Aleuritic-pelitic mud }\end{array}$ & $\begin{array}{r}16.4 \\
20.6 \\
10.8 \\
12.0 \\
9.8 \\
47.8 \\
3.2 \\
45.8 \\
18.7 \\
4.0 \\
4.1 \\
1.6 \\
0.8 \\
0.1 \\
2.0 \\
\end{array}$ & $\begin{array}{r}10.5 \\
13.0 \\
8.8 \\
9.1 \\
7.1 \\
31.0 \\
4.5 \\
27.2 \\
11.1 \\
6.4 \\
2.9 \\
0.5 \\
0.7 \\
0.3 \\
1.5 \\
\end{array}$ & $\begin{array}{l}21.1 \\
24.3 \\
22.8 \\
21.1 \\
22.7 \\
13.8 \\
56.3 \\
15.7 \\
18.8 \\
25.0 \\
37.4 \\
46.4 \\
30.7 \\
48.7 \\
31.9 \\
\end{array}$ & $\begin{array}{r}13.7 \\
5.0 \\
7.2 \\
9.4 \\
14.5 \\
1.5 \\
10.0 \\
4.3 \\
10.0 \\
28.6 \\
18.6 \\
- \\
21.6 \\
22.8 \\
9.3\end{array}$ & $\begin{array}{r}30.1 \\
24.8 \\
36.0 \\
23.5 \\
33.9 \\
2.9 \\
14.0 \\
2.8 \\
23.2 \\
24.7 \\
18.6 \\
- \\
29.8 \\
12.7 \\
23.1\end{array}$ & $\begin{array}{r}8.2 \\
12.4 \\
14.3 \\
25.0 \\
12.1 \\
2.9 \\
12.0 \\
4.2 \\
18.2 \\
11.4 \\
18.5 \\
- \\
16.3 \\
15.3 \\
32.3\end{array}$ & $\begin{array}{r}52.0 \\
42.2 \\
57.5 \\
57.9 \\
60.5 \\
7.3 \\
36.0 \\
11.3 \\
51.4 \\
64.7 \\
55.7 \\
51.6 \\
67.7 \\
50.8 \\
64.7 \\
\end{array}$ \\
\hline
\end{tabular}

aFrom sea bed.

b According to Russian classification. 
TABLE 2

Mineral Composition of Coarse-Silty Fraction $(\mathbf{0 . 1}-\mathbf{0 . 0 5} \mathrm{mm})$ of Sediments of the Vøring Plateau, DSDP Leg 38

\begin{tabular}{|c|c|c|c|c|c|c|c|c|c|c|c|c|c|c|c|c|c|c|c|}
\hline & & \multicolumn{18}{|c|}{ Heavy Fraction $(\%)^{a}$} \\
\hline \multirow[b]{2}{*}{$\begin{array}{l}\text { Sample } \\
\text { (Interval } \\
\text { in } \mathrm{cm} \text { ) }\end{array}$} & \multirow[b]{2}{*}{$\begin{array}{c}\text { Depth } \\
\text { (m) }\end{array}$} & \multicolumn{18}{|c|}{ Volcano-terrigenous } \\
\hline & & $\begin{array}{c}\text { Content } \\
\text { Heavy } \\
\text { Fraction }(\%)\end{array}$ & 冚 & 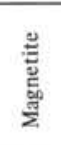 & 冚 & 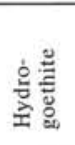 & 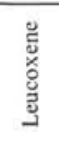 & $\frac{\frac{D}{\frac{D}{5}}}{\frac{D}{0}}$ & 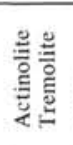 & 吾总 & 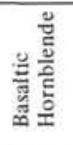 & 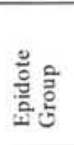 & 预 & 氖 & 产 & कू & 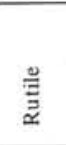 & 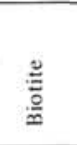 & 를 \\
\hline \multicolumn{20}{|l|}{ Site 339} \\
\hline $2-1.119-121$ & 9.2 & 1.2 & - & 1.4 & 13.0 & 1.4 & 0.3 & 23.0 & 0.3 & - & - & 29.8 & 5.3 & 2.4 & 2.7 & 0.3 & 0.8 & 1.0 & 0.3 \\
\hline $2-3,129-131$ & 12.4 & 2.9 & - & - & 4.3 & 0.4 & 0.7 & 21.7 & 0.4 & - & - & 10.9 & 1.1 & 1.4 & 1.4 & - & - & 0.7 & 0.4 \\
\hline $3-4,142-144$ & 23.7 & 1.3 & - & 0.7 & 24.8 & 0.4 & - & 4.0 & - & - & 0.4 & 11.0 & 12.0 & 3.5 & 1.8 & 0.4 & 1.1 & - & - \\
\hline $4-2,139-140$ & 38.0 & 1.9 & - & 0.7 & 24.8 & 3.8 & 1.4 & 19.6 & - & - & - & 23.8 & 8.0 & 2.8 & 1.4 & - & 0.7 & 1.0 & 0.4 \\
\hline $5-1.139-140$ & 38.0 & 1.9 & - & 0.4 & 8.9 & - & 0.4 & 32.1 & 0.4 & - & - & 21.4 & 7.9 & 2.1 & 1.1 & 1.4 & 0.7 & 0.4 & 0.4 \\
\hline $6-3,102-104$ & 49.9 & 1.2 & - & 0.7 & 13.6 & - & - & 26.0 & 0.4 & 0.3 & - & 21.6 & 13.2 & 1.8 & 0.4 & - & 0.3 & 0.4 & - \\
\hline $7-1,130-132$ & 56.9 & 0.9 & - & 3.9 & 9.4 & - & 3.5 & 23.0 & 0.7 & - & 0.4 & 23.0 & 7.0 & 2.3 & 1.6 & - & 1.6 & 0.4 & - \\
\hline $8-1.100-102$ & 66.0 & 2.6 & - & - & 8.0 & 0.3 & 0.3 & 40.5 & - & 0.7 & - & 17.9 & 12.3 & 2.7 & 2.7 & 0.3 & 0.7 & 2.0 & 0.3 \\
\hline $10-3,70-73$ & 87.9 & 0.2 & - & 1.3 & 10.5 & 2.6 & - & 1.3 & - & - & - & 7.9 & 7.9 & 14.5 & - & - & 2.6 & - & - \\
\hline $12-2,70-72$ & 104.2 & 2.3 & - & 1.9 & 0.9 & - & 0.9 & 2.8 & - & - & - & 19.4 & 11.1 & 7.4 & - & 1.9 & 2.8 & - & 1.9 \\
\hline Site 340 & & & & & & & & & & & & & & & & & & & \\
\hline $1, \mathrm{CC}$ & 9.5 & 1.9 & 0.7 & 0.3 & 16.3 & 5.2 & 0.7 & 19.4 & 1.1 & 1.4 & 0.7 & 19.4 & 6.9 & 4.5 & 1.7 & - & 0.7 & 0.4 & - \\
\hline $2-1,38-40$ & 9.9 & 1.5 & 0.3 & 2.0 & 11.6 & 2.1 & 3.8 & 16.4 & 0.7 & 0.3 & 0.7 & 34.8 & 6.1 & 3.1 & 2.4 & 1.0 & 0.3 & 1.0 & 0.6 \\
\hline $2-3,75-77$ & 13.5 & 0.2 & -- & 1.5 & 1.5 & 1.5 & 1.5 & 3.1 & - & 1.5 & - & 9.2 & 4.6 & 3.1 & - & - & - & - & - \\
\hline $3-3,75-77$ & 23.0 & 1.8 & - & + & $+b$ & $++\mathrm{c}$ & - & + & - & - & - & + & + & + & - & - & - & - & - \\
\hline $4-4,70-72$ & 40.3 & 0.1 & - & - & - & - & - & 4.0 & - & - & - & - & 5.9 & - & - & - & - & 7.8 & 2.0 \\
\hline $5-2,70-72$ & 40.3 & 0.1 & - & - & + & + & - & + & - & - & - & + & + & ++ & - & - & - & - & - \\
\hline $6-3,60-62$ & 51.3 & 0.9 & - & - & 2.6 & 78.2 & - & - & - & - & - & - & 1.3 & 5.1 & - & - & - & - & - \\
\hline $7-1,95-97$ & 58.0 & 0.5 & - & 5.3 & 1.1 & 20.2 & 1.1 & 3.2 & - & - & - & 6.4 & 4.2 & 3.2 & - & - & 1.1 & 1.1 & 1.1 \\
\hline $7-6,60-62$ & 65.0 & 0.3 & - & - & 4.5 & - & - & 7.6 & - & - & - & 9.1 & 3.0 & 6.1 & - & - & 1.5 & - & - \\
\hline $8-4,72-74$ & 72.0 & 0.9 & - & 1.1 & 3.2 & - & - & 19.1 & - & - & 2.1 & 3.2 & - & 39.3 & 7.4 & - & 1.1 & - & 1.1 \\
\hline $9-3 \cdot 70-72$ & 79.9 & 0.2 & - & 1.9 & 5.7 & - & 1.9 & 3.8 & - & - & - & 1.9 & - & 5.8 & - & - & 1.9 & - & 1.9 \\
\hline $10-2,50-52$ & 87.6 & 0.1 & - & - & 1.7 & 3.4 & - & - & - & - & - & - & 3.4 & 5.2 & 1.7 & - & - & 3.4 & 5.2 \\
\hline $11-4,81-83$ & 100.7 & 0.3 & - & - & - & 1.6 & - & 4.7 & - & - & - & - & - & - & - & - & - & 3.2 & 4.7 \\
\hline Site 341 & & & & & & & & & & & & & & & & & & & \\
\hline $1-3.75 .77$ & 3.4 & 1.2 & 0.7 & - & 11.6 & 0.7 & 1.8 & 28.8 & 2.0 & - & 0.7 & 22.1 & 13.3 & 3.2 & 1.4 & 1.0 & 0.7 & 1.4 & 1.8 \\
\hline $2-2,75-77$ & 11.9 & 2.0 & - & - & 8.7 & 1.4 & 0.7 & 26.5 & 2.4 & 0.7 & - & 26.9 & 7.4 & 2.4 & 2.1 & 0.4 & 0.4 & 12.9 & - \\
\hline $4-1,75-77$ & 29.3 & 3.4 & - & - & 4.2 & 2.5 & 3.0 & 29.8 & 0.8 & 0.4 & - & 27.2 & 4.9 & 1.1 & 1.9 & 1.5 & 0.8 & 5.6 & 0.8 \\
\hline $6-3,50$ & 51.25 & 0.2 & - & - & - & - & - & 4.9 & - & - & - & 2.8 & 2.1 & 2.1 & - & - & - & - & 3.5 \\
\hline $7-3,50$ & 60.8 & 1.6 & - & - & 1.0 & 0.7 & 0.7 & 17.7 & - & - & - & 9.4 & 5.9 & 2.4 & 0.4 & - & 1.0 & 0.4 & - \\
\hline $8-3,50$ & 70.3 & 5.6 & - & - & 3.3 & 0.7 & 0.4 & 33.5 & 2.2 & - & - & 30.1 & 13.4 & 1.5 & 1.1 & 0.4 & 1.9 & 2.6 & - \\
\hline $10-1,109-111$ & 86.7 & 6.3 & - & - & 4.4 & - & 0.7 & 41.4 & 1.3 & 0.4 & 0.4 & 28.4 & 11.6 & 1.7 & 0.4 & 0.4 & 0.7 & 0.4 & - \\
\hline $13-1,142-144$ & 107.0 & 6.4 & 0.7 & - & 1.5 & 2.6 & - & 43.4 & 1.2 & - & - & 20.8 & 6.2 & 0.4 & 1.5 & - & - & 4.4 & - \\
\hline $17-2,129-131$ & 183.5 & 8.7 & - & - & 3.0 & - & 0.8 & 34.2 & - & - & - & 17.3 & 9.0 & 2.6 & 1.9 & - & 0.8 & 9.4 & 3.8 \\
\hline $20-2,100-102$ & 240.2 & 1.3 & - & - & 0.3 & - & - & 5.5 & - & 0.3 & - & 3.7 & 1.4 & 1.1 & 0.7 & - & 0.3 & 0.7 & 1.1 \\
\hline $21-4,90-92$ & 262.3 & 3.2 & 0.7 & - & 1.4 & 1.0 & 0.8 & 28.3 & 0.3 & 0.3 & 0.3 & 15.5 & 3.1 & 0.3 & 1.7 & 0.3 & - & 0.7 & 0.7 \\
\hline $28-3,100-103$ & 393.9 & 19.0 & - & - & - & - & - & 0.3 & - & - & - & - & 0.3 & - & - & - & - & 0.3 & 0.3 \\
\hline $29-4.118-120$ & 405.1 & 19.5 & - & - & - & - & - & - & - & - & - & - & - & - & - & - & - & - & - \\
\hline $30-4,41-43$ & 413.7 & 9.1 & - & - & - & - & - & - & - & - & - & 0.4 & 0.7 & - & - & - & - & - & - \\
\hline $324,50-52$ & 432.9 & 9.8 & - & - & - & - & - & 0.6 & - & - & - & 0.3 & 0.3 & - & 0.3 & - & - & - & - \\
\hline Site 342 & & & & & & & & & & & & & & & & & & & \\
\hline $1-2,49-51$ & 2.0 & 1.4 & 1.0 & - & 12.5 & 0.3 & 2.8 & 25.6 & - & - & 0.3 & 27.0 & 13.8 & 2.1 & 3.8 & - & 0.7 & 0.7 & - \\
\hline $1-5,69-71$ & 6.7 & 1.6 & - & - & 12.8 & 2.4 & 2.7 & 23.2 & 1.0 & 0.3 & - & 26.8 & 9.7 & 3.7 & 1.7 & - & 0.7 & 0.3 & - \\
\hline $2-2,94-96$ & 40.1 & 1.6 & - & - & 5.0 & 2.0 & 1.0 & 18.1 & 0.6 & 0.7 & 0.3 & 23.7 & 9.4 & 1.0 & 1.3 & - & - & 0.3 & 0.3 \\
\hline $3-4,100-102$ & 90.9 & 1.7 & - & - & - & - & - & 0.7 & - & - & - & 0.3 & 0.3 & - & - & - & - & - & 1.0 \\
\hline $4-2,110-112$ & 125.8 & 0.3 & - & - & - & - & - & 0.8 & - & - & - & 0.8 & - & - & - & - & - & 1.5 & 0.8 \\
\hline $5-2,100-102$ & 135.2 & 0.1 & - & 0.9 & - & 2.7 & - & 0.9 & - & - & - & 0.9 & - & - & - & - & - & - & 0.9 \\
\hline $5-6,100-102$ & 141.5 & 0.2 & - & 0.8 & - & 1.6 & - & - & - & - & - & - & 0.8 & - & - & - & - & - & 2.4 \\
\hline $6-4,61-62$ & 147.5 & 2.5 & - & - & - & - & - & 0.7 & - & - & - & 1.1 & - & 0.4 & - & - & - & - & 0.4 \\
\hline Site 343 & & & & & & & & & & & & & & & & & & & \\
\hline $1-1,75-77$ & 0.2 & 1.1 & 0.7 & - & 8.6 & 2.1 & 3.8 & 30.7 & 0.7 & 0.3 & - & 23.1 & 11.7 & 2.8 & 3.4 & - & 0.7 & 0.7 & 0.3 \\
\hline $1-3,75-77$ & 1.2 & 1.3 & 0.7 & 0.3 & 7.5 & 4.1 & 3.4 & 29.4 & 0.7 & - & - & 22.2 & 11.9 & 3.8 & 1.7 & - & 0.6 & 1.4 & 0.3 \\
\hline $2-2,105-107$ & 5.7 & 1.1 & - & 0.7 & 12.4 & 2.7 & 2.7 & 17.8 & - & - & - & 16.8 & 16.9 & 3.0 & 1.7 & - & 1.0 & 1.3 & 3.0 \\
\hline $2-4,75-77$ & 8.7 & 1.4 & 1.4 & 1.1 & 10.6 & 1.8 & 2.1 & 17.3 & 0.4 & 0.7 & - & 15.5 & 18.8 & 2.8 & 1.8 & - & 0.4 & - & 0.4 \\
\hline $2, \mathrm{CC}$ & 12.5 & 2.2 & - & - & 13.9 & 4.1 & 3.7 & 15.6 & 0.3 & - & - & 23.8 & 16.0 & 2.4 & 1.4 & 0.3 & 0.3 & 1.4 & 0.3 \\
\hline $3-2,129-131$ & 53.5 & 2.8 & - & 1.4 & 5.1 & 0.3 & 3.0 & 24.0 & - & 1.0 & 1.0 & 19.9 & 16.9 & 3.0 & 2.0 & 0.7 & 0.3 & - & 0.7 \\
\hline $3-4,129-131$ & 56.0 & 1.7 & - & - & 5.4 & - & - & 7.0 & - & - & - & 4.7 & 5.7 & 1.0 & 0.3 & - & 0.3 & 17.4 & 17.8 \\
\hline $3-6,119-121$ & 59.7 & 4.4 & 1.0 & 0.3 & 8.5 & 1.4 & 0.5 & 25.8 & 0.7 & 0.3 & 0.3 & 25.8 & 15.6 & 0.3 & 1.4 & 0.7 & 0.7 & 0.3 & - \\
\hline $4-3,110-112$ & 102.6 & 1.6 & - & 0.4 & 14.2 & 2.1 & 1.4 & 33.0 & 1.8 & - & 0.3 & 18.1 & 15.2 & 1.4 & 1.8 & - & 0.7 & 0.4 & 0.7 \\
\hline $5-2,19-21$ & 147.3 & 0.9 & 1.5 & 0.4 & 23.0 & 10.9 & 2.6 & 8.0 & - & 0.4 & - & 19.7 & 16.0 & 10.9 & 2.6 & - & 0.4 & - & - \\
\hline $7-0,30-32$ & 202.0 & 0.7 & - & 3.1 & 0.3 & 1.0 & 0.3 & - & - & - & - & - & 0.3 & 0.3 & 0.3 & - & - & 1.0 & 1.3 \\
\hline $8-3,100-103$ & 216.3 & 30.3 & - & - & - & - & - & - & - & - & - & 0.4 & - & - & - & - & - & 2.9 & 0.7 \\
\hline $11-2,130-132$ & 243.5 & 1.2 & - & 0.7 & - & 0.3 & - & - & - & - & - & 0.3 & - & - & - & - & - & - & 4.7 \\
\hline $15-1,99-101$ & 270.1 & 3.2 & - & 2.2 & - & - & - & 0.4 & - & - & - & 0.4 & 0.5 & 0.4 & - & - & - & - & 8.0 \\
\hline $16-1,18-20$ & 282.0 & 0.9 & - & 1.1 & - & 1.1 & - & 1.8 & 0.4 & - & - & 1.1 & - & - & - & - & - & - & 7.2 \\
\hline
\end{tabular}

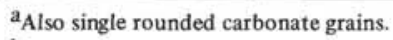

bSingle grains.

$\mathrm{c}_{\text {Abundant. }}$ 
TABLE 2 - Continued

\begin{tabular}{|c|c|c|c|c|c|c|c|c|c|c|c|c|c|c|c|c|c|c|c|c|}
\hline \multicolumn{21}{|c|}{ Heavy Fraction $(\%)^{\mathrm{a}}$} \\
\hline \multicolumn{14}{|c|}{ Volcano-terrigenous } & \multirow{2}{*}{ 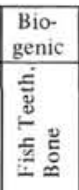 } & \multicolumn{6}{|l|}{1} \\
\hline 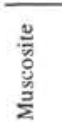 & 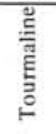 & 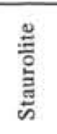 & 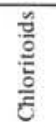 & 華 & 충 & 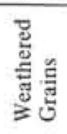 & 总 & 总 & 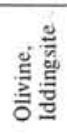 & 莺 & 言参 & 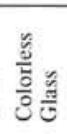 & 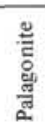 & & 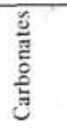 & $\stackrel{\Xi}{\underline{z}}$ & 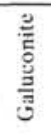 & 旅 & 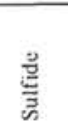 & $\frac{\overline{3}}{\frac{\overline{3}}{0}}$ \\
\hline- & 0.3 & - & - & 0.3 & 1.1 & 0.3 & 4.0 & 1.0 & - & 4.7 & - & - & - & - & $0.3^{\mathrm{a}}$ & - & - & 0.3 & 4.7 & 99.9 \\
\hline 0.4 & - & - & - & - & 1.5 & - & 5.4 & - & - & 1.8 & - & - & - & - & 0.4 & - & - & - & 47.1 & 100.0 \\
\hline 0.4 & 0.4 & 0.4 & - & - & - & - & 15.2 & 0.7 & - & - & - & - & - & - & 1.4 & - & - & - & 21.3 & 99.9 \\
\hline- & 2.1 & - & - & - & - & - & 1.0 & 1.1 & 0.4 & 1.1 & - & - & - & - & 3.5 & - & - & - & 2,4 & 100.0 \\
\hline - & 1.4 & - & - & 0.4 & - & - & 7.5 & 2.5 & 0.4 & 1.1 & - & - & - & - & 7.1 & - & - & - & 2.1 & 100.1 \\
\hline 0.4 & 0.3 & 0.3 & - & 0.4 & 0.9 & - & 1.1 & 0.4 & - & 1.1 & - & - & - & - & 9.9 & - & - & - & 6.2 & 99.7 \\
\hline- & - & 0.4 & 0.4 & 0.4 & - & - & 2.3 & 0.4 & - & 2.3 & 0.4 & - & - & 0.4 & 0.8 & - & - & - & 15.6 & 100.2 \\
\hline 1.0 & - & 0.3 & - & 0.3 & - & - & 4.0 & 1.0 & - & 0.7 & - & - & - & - & 1.0 & - & - & - & 3.0 & 100.0 \\
\hline- & - & - & - & - & - & - & 1.3 & - & - & - & - & - & - & - & 5.3 & - & - & - & 44.7 & 99.9 \\
\hline- & - & - & - & - & - & 1.9 & 1.9 & - & - & - & 5.5 & 2.8 & - & - & - & - & - & - & 37.0 & 100.1 \\
\hline- & 0.7 & - & - & - & - & - & 4.2 & 0.7 & 0.4 & 2.1 & - & - & - & - & 1.7 & 10.1 & - & - & 0.7 & 100.0 \\
\hline 1.0 & - & 0.3 & - & - & 1.7 & - & 2.0 & - & - & 1.0 & - & - & - & - & 2.0 & 0.7 & - & - & 3.8 & 99.7 \\
\hline 49.2 & - & - & - & - & 1.5 & - & 1.5 & - & - & 1.5 & - & 1.5 & - & 4.6 & 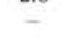 & 1.5 & - & - & 10.8 & 99.6 \\
\hline+ & - & - & - & - & - & - & - & - & - & - & - & + & - & - & + & - & - & - & ++ & - \\
\hline 3.9 & - & - & - & - & 2.0 & - & 3.9 & - & - & 3.9 & - & 2.0 & - & 2.0 & - & - & - & - & 62.7 & 100.1 \\
\hline+ & - & - & - & - & - & - & + & - & - & - & - & - & - & - & + & - & - & - & ++ & - \\
\hline- & - & - & - & - & - & - & - & - & - & - & 1.3 & 1.3 & - & 2.6 & - & - & - & - & 7.7 & 100.1 \\
\hline 2.2 & - & - & - & 1.1 & - & 23.4 & 1.1 & - & - & 6.3 & 4.2 & 2.1 & - & 4.3 & - & 1.1 & - & - & 6.4 & 100.2 \\
\hline 6.1 & - & - & - & - & - & - & 4.5 & - & - & - & - & 1.5 & - & - & - & - & - & - & 56.1 & 100.0 \\
\hline- & - & - & - & - & - & - & 1.1 & - & - & 10.6 & 2.1 & 1.1 & - & 2.2 & - & - & - & - & 5.3 & 100.0 \\
\hline 1.9 & - & - & - & - & - & 5.8 & 5.8 & - & - & - & - & 1.9 & - & 1.9 & - & - & - & - & 57.7 & 99.8 \\
\hline 1.7 & - & - & - & - & - & 5.2 & - & 1.7 & - & 3.4 & 3.4 & 3.4 & - & 5.2 & - & - & - & - & 51.7 & 99.7 \\
\hline- & - & - & - & - & 1.7 & 3.2 & - & - & 3.1 & 3.1 & 1.6 & - & - & 1.6 & - & - & - & - & 70.3 & 100.2 \\
\hline 1.0 & 1.4 & - & - & - & 0.4 & - & 0.7 & 1.4 & - & 2.8 & - & - & - & - & 1.0 & - & - & - & - & 99.9 \\
\hline- & - & - & - & - & - & - & 2.1 & 1.4 & - & - & - & - & - & - & 0.4 & - & - & - & 3.5 & 100.3 \\
\hline 0.4 & 0.4 & - & 0.4 & 0.4 & - & - & 3.8 & 0.7 & - & 5.7 & - & - & - & - & 3.0 & 0.4 & 0.4 & - & 0.4 & 100.3 \\
\hline 0.7 & 0.7 & - & - & - & - & - & 0.7 & 0.7 & - & - & - & 2.1 & - & - & - & - & - & - & 79.9 & 100.2 \\
\hline- & - & - & - & - & - & - & 0.7 & 0.4 & - & 0.4 & 0.4 & 0.4 & - & - & 1.4 & - & - & - & 56.9 & 100.2 \\
\hline 0.7 & 0.7 & - & - & 0.4 & - & - & - & - & 0.4 & 0.4 & - & - & - & - & 3.0 & - & - & - & 3.3 & 100.0 \\
\hline- & 0.7 & 0.4 & - & - & 0.4 & - & 1.7 & 0.7 & - & 1.0 & - & - & - & - & 2.0 & - & - & - & 1.0 & 100.1 \\
\hline- & 0.7 & 0.4 & - & - & - & - & - & 0.7 & - & 1.9 & - & - & - & - & 3.9 & - & - & - & 5.1 & 100.1 \\
\hline 0.8 & 0.8 & 0.4 & - & - & - & - & 8.6 & - & - & 1.1 & - & - & - & - & 1.5 & - & - & - & 3.8 & 99.8 \\
\hline 0.3 & 0.3 & $\cdots$ & - & - & 0.3 & - & 0.7 & 0.3 & - & - & - & - & - & - & 0.3 & - & - & - & 83.0 & 100.3 \\
\hline 0.8 & - & 0.3 & 0.3 & - & 0.3 & - & 1.0 & - & - & - & - & - & - & - & 0.9 & - & - & - & 40.7 & 99.6 \\
\hline 0.7 & - & - & - & - & - & - & - & - & - & - & 2.7 & 0.3 & - & - & - & - & - & - & 94.9 & 99.8 \\
\hline- & - & - & - & - & - & - & - & - & - & - & 1.0 & - & - & - & 0.3 & - & - & - & 98.6 & 99.9 \\
\hline- & - & - & - & - & - & - & - & - & - & - & 1.1 & 0.7 & - & - & - & - & - & - & 97.1 & 100.0 \\
\hline - & - & - & - & - & - & - & - & - & - & - & 4.1 & 0.3 & - & - & - & - & - & - & 93.6 & 100.0 \\
\hline - & 1.7 & 0.7 & - & - & 0.6 & 0.3 & 3.4 & 0.3 & 0.3 & 1.4 & - & - & - & - & - & - & - & 0.3 & - & 99.6 \\
\hline - & 0.7 & 0.3 & - & - & 0.7 & - & 4.7 & 0.3 & - & $\begin{array}{l}6.4 \\
6.4\end{array}$ & - & - & - & - & 1.7 & - & - & - & - & 100.1 \\
\hline- & 0.7 & - & - & - & 2.1 & 0.3 & 2.3 & - & - & 0.7 & - & - & - & - & 0.3 & - & - & - & 30.0 & 100.1 \\
\hline 1.3 & - & - & - & - & - & - & - & - & - & - & 1.0 & 1.0 & - & 0.7 & - & - & - & - & 93.7 & 100.0 \\
\hline 1.5 & - & - & - & - & - & - & - & - & - & - & 6.0 & 0.4 & - & - & - & - & - & - & 88.3 & 100.1 \\
\hline 5.4 & - & - & - & - & - & - & - & - & - & 0.9 & 6.2 & 0.9 & - & 0.9 & - & - & - & - & 79.5 & 100.1 \\
\hline- & - & - & - & - & - & - & - & 0.8 & - & - & 4.1 & 0.8 & 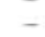 & - & 0.8 & - & - & - & 87.8 & 99.9 \\
\hline 0.4 & - & - & - & - & - & - & 0.4 & - & - & - & 0.4 & - & - & 11 & 0.4 & - & - & - & 94.9 & 100.2 \\
\hline- & 1.0 & - & 0.3 & - & - & - & 4.1 & 0.3 & - & 3.8 & - & - & - & 0.3 & 0.3 & - & - & - & - & 99.7 \\
\hline 0.7 & 1.0 & 0.3 & $\because$ & - & - & - & 2.0 & - & - & 5.5 & - & - & - & 0.3 & 1.0 & - & - & 1.0 & - & 99.8 \\
\hline 1.3 & 0.3 & - & - & 0.3 & - & 2.7 & 3.4 & - & - & 1.4 & - & - & - & - & 2.7 & - & - & - & 0.3 & 99.7 \\
\hline- & 1.4 & - & - & - & 1.6 & 10.4 & 2.4 & - & - & 0.7 & - & - & - & - & 4.3 & - & - & - & 9.5 & 100.3 \\
\hline 0.7 & 0.7 & - & - & - & 1.1 & 2.8 & 3.2 & 1.1 & - & 1.8 & - & - & - & - & 4.1 & - & - & - & 3.1 & 99.9 \\
\hline- & 0.7 & 0.3 & - & 0.3 & - & 1.0 & 3.4 & 1.4 & - & 0.7 & - & - & - & - & 8.1 & - & - & - & 4.7 & 99.9 \\
\hline 29.2 & 0.3 & - & - & - & - & - & 0.7 & 0.7 & - & - & - & - & - & - & 5.7 & - & - & - & 3.7 & 99.9 \\
\hline & 1.4 & 0.3 & - & - & - & - & 2.4 & 1.0 & - & - & - & - & - & - & 8.9 & - & - & - & 3.0 & 100.0 \\
\hline 0.7 & 1.4 & 0.4 & - & 0.3 & 0.7 & - & 3.2 & 0.4 & - & - & - & - & - & - & 0.3 & - & - & - & 0.7 & 99.6 \\
\hline- & 0.4 & - & 0.4 & - & - & - & 2.2 & - & - & 0.7 & - & - & - & - & - & - & - & - & - & 100.1 \\
\hline 77.2 & - & - & - & - & - & - & 0.3 & - & - & - & 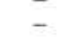 & 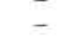 & 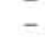 & 0.3 & - & - & - & 0.7 & 13.3 & 99.7 \\
\hline 0.7 & - & - & - & - & - & - & - & - & - & - & - & - & - & - & 63.9 & - & - & - & 31.4 & 100.0 \\
\hline 1.4 & - & - & - & - & - & 4.4 & - & - & - & - & - & - & - & - & - & - & - & - & 88.1 & 99.9 \\
\hline 16.8 & - & - & - & - & - & 2.2 & - & - & - & - & - & - & - & - & - & - & - & - & 69.0 & 99.9 \\
\hline 12.3 & - & - & 0.7 & - & - & $\because$ & - & - & - & - & - & - & - & 1.8 & - & - & - & - & 72.4 & 99.9 \\
\hline
\end{tabular}


TABLE 2 - Continued

\begin{tabular}{|c|c|c|c|c|c|c|c|c|c|c|c|c|c|c|c|c|c|c|c|c|c|c|}
\hline \multirow[b]{3}{*}{$\begin{array}{l}\text { Sample } \\
\text { (Interval } \\
\text { in } \mathrm{cm} \text { ) }\end{array}$} & \multirow[b]{3}{*}{$\begin{array}{c}\text { Depth } \\
\text { (m) }\end{array}$} & \multicolumn{21}{|c|}{ Light Fraction (\%) } \\
\hline & & \multicolumn{7}{|c|}{ Terrigenous } & & Volca & logenic & & & & Biog & & & & & uthigen & & \\
\hline & & $\frac{y}{3}$ & 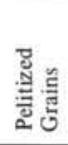 & 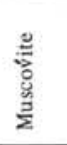 & 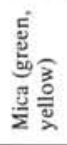 & 商 & 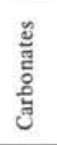 & 总 & 言参 & 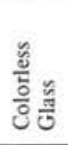 & 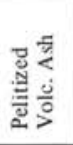 & 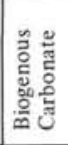 & 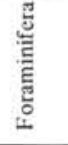 & $\begin{array}{l}\frac{\tilde{u}}{3} \\
\frac{\tilde{y}}{2} \\
\frac{1}{n}\end{array}$ & $\hat{\mathrm{g}}$ & 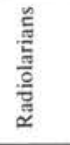 & 总 & 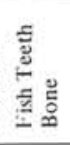 & 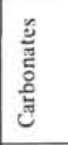 & 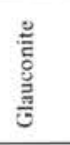 & 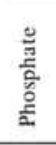 & 氖 \\
\hline Site 339 & & & & & & & & & & & & & & & & & & & & & & \\
\hline $2-1,119-121$ & 9.2 & 69.4 & 12.0 & - & 1.4 & 7.0 & & - & - & - & - & 0.7 & & - & & - & - & 0.4 & 2.5 & & - & 100.1 \\
\hline $2-3,129-131$ & 12.4 & $\begin{array}{l}76.4 \\
64.1\end{array}$ & $\begin{array}{r}8.5 \\
12.3\end{array}$ & - & 0.3 & $\begin{array}{r}7.2 \\
19.5\end{array}$ & $\overline{0.7}$ & - & - & 0.3 & - & - & 3.8 & - & 0.3 & - & - & - & 0.4 & $\begin{array}{l}0.3 \\
0.7\end{array}$ & - & $\begin{array}{l}99.8 \\
99.9\end{array}$ \\
\hline $\begin{array}{l}3-4,142-144 \\
4-2,121-123\end{array}$ & $\begin{array}{l}23.7 \\
33.0\end{array}$ & $\begin{array}{l}64.1 \\
78.9\end{array}$ & $\begin{array}{r}12.3 \\
2.1\end{array}$ & - & $\begin{array}{l}0.4 \\
1.8\end{array}$ & $\begin{array}{l}19.5 \\
12.3\end{array}$ & 0.7 & - & - & $=$ & - & - & 1.8 & $\overline{0.4}$ & - & & & & $\begin{array}{l}0.4 \\
0.7\end{array}$ & $\begin{array}{l}0.1 \\
1.1\end{array}$ & $=$ & $\begin{array}{r}99.9 \\
100.2\end{array}$ \\
\hline $\begin{array}{l}4-2,121-123 \\
5-1,139-140\end{array}$ & $\begin{array}{l}33.0 \\
38.0\end{array}$ & $\begin{array}{l}78.9 \\
64.3\end{array}$ & $\begin{array}{l}2.1 \\
6.7\end{array}$ & - & $\begin{array}{l}1.8 \\
2.4\end{array}$ & $\begin{array}{l}12.3 \\
17.8\end{array}$ & $\overline{-}$ & - & - & 0.7 & - & - & 1.8 & 0.4 & 5 & 1.0 & 0.4 & - & 1.0 & 3.7 & 0.3 & $\begin{array}{r}100.2 \\
99.9\end{array}$ \\
\hline $\begin{array}{l}5-1,139-140 \\
6-3,102-104\end{array}$ & $\begin{array}{l}38.0 \\
49.9\end{array}$ & 64.8 & $\begin{array}{l}0.1 \\
9.2\end{array}$ & $\begin{array}{l}1.0 \\
0.4\end{array}$ & $\begin{array}{l}2.4 \\
0.7\end{array}$ & 17.0 & - & - & $\overline{-}$ & $\overline{0.4}$ & $\overline{-}$ & - & $\begin{array}{l}0.7 \\
0.4\end{array}$ & $=$ & $\begin{array}{l}1.0 \\
0.7\end{array}$ & 0.7 & - & - & 0.7 & 4.8 & - & 99.8 \\
\hline $7-1 \quad 130-132$ & 56.9 & 6.0 & 9.0 & - & 0.3 & 5.7 & 0.7 & - & - & 17.7 & - & - & 0.3 & - & 42.8 & 17.4 & - & - & - & - & - & 99.9 \\
\hline $8-1,100-102$ & 66.0 & 68.6 & 3.4 & 0.3 & 0.7 & 20.7 & 0.3 & - & - & - & - & - & 1.0 & 0.3 & 1.7 & 0.7 & - & - & 0.7 & 1.4 & 0.3 & 100.1 \\
\hline $10-3.70-73$ & 87.9 & - & - & - & 0.3 & - & - & - & - & 4.0 & - & - & - & 2.7 & 84.8 & 8.2 & - & - & - & - & - & 100.0 \\
\hline $12-2,70-72$ & 104.2 & 2.7 & 2.1 & - & - & 1.7 & - & - & 1.7 & 9.2 & - & - & - & 2.7 & 41.4 & 38.4 & - & & - & - & - & 99.9 \\
\hline Site 340 & & & & & & & & & & & & & & & & & & & & & & \\
\hline $1, \mathrm{CC}$ & 9.5 & 66.6 & 5.2 & - & 0.3 & 10.3 & 2.1 & - & - & 1.0 & - & - & 3.8 & $=$ & 6.2 & 2.4 & - & - & 1.4 & 0.7 & - & 100.0 \\
\hline $2-1,38-40$ & 9.9 & 29.2 & 6.5 & - & 0.4 & 1.8 & 0.7 & - & - & 2.5 & - & - & - & 0.7 & 40.4 & 17.0 & - & - & - & 0.7 & - & 99.9 \\
\hline $2-3,75-77$ & 13.5 & 0.3 & - & - & - & - & - & - & - & 4.2 & 11.0 & - & - & - & 71.9 & 12.8 & - & - & - & - & - & 100.2 \\
\hline $3-3,75-77$ & 23.0 & 1.5 & - & - & - & 0.4 & - & - & 0.3 & 8.0 & 17.3 & - & - & 1.1 & 13.2 & 58.2 & - & - & - & - & - & 100.0 \\
\hline $4-4,75-77$ & 34.0 & 0.7 & - & - & 0.8 & - & - & - & 0.4 & 17.1 & 7.8 & - & - & 2.8 & 8.9 & 61.6 & - & - & - & - & - & 100.1 \\
\hline $5-2,70-72$ & 40.3 & - & - & - & 0.4 & 1.5 & - & - & - & 11.1 & 21.5 & - & - & 3.0 & 8.9 & 53.7 & - & - & - & - & - & 100.1 \\
\hline $6-3,60-62$ & 51.3 & - & 0.3 & - & - & - & - & - & - & 5.6 & 3.6 & - & - & - & 75.7 & 14.6 & - & - & - & - & - & 99.8 \\
\hline $7-1,95-97$ & 58.0 & - & - & - & 0.3 & 0.3 & - & - & - & 5.8 & 50.0 & - & - & - & 21.4 & $19: 3$ & - & - & - & - & - & 100.1 \\
\hline $7-6,95-97$ & 65.0 & 0.4 & - & - & - & - & - & $=$ & - & 1.5 & 6.3 & - & - & 0.7 & 74.6 & 16.5 & - & - & - & - & - & 100.0 \\
\hline $8-4,72-74$ & 72.0 & 0.4 & - & - & 0.4 & 0.4 & - & - & 2.1 & 16.0 & 10.7 & - & - & 3.9 & 14.2 & 52.0 & - & - & - & - & - & 100.1 \\
\hline $9-3,70-72$ & 79.9 & - & - & - & - & 0.4 & - & - & 0.7 & 3.3 & - & - & - & - & 17.8 & 77.8 & - & - & - & - & - & 100.0 \\
\hline $10-2,50-52$ & 81.6 & - & - & - & - & 0.4 & - & - & - & 5.5 & 2.6 & - & - & - & 11.3 & 80.3 & - & - & - & - & - & 100.1 \\
\hline $11-4,81-83$ & 100.7 & - & - & - & 0.4 & - & - & - & 0.7 & 3.0 & - & - & - & - & - & 95.9 & - & & - & - & - & 100.0 \\
\hline Site 341 & & & & & & & & & & & & & & & & & & & & & & \\
\hline $1-3,75-77$ & 3.4 & 74.6 & - & - & 0.6 & 18.8 & - & - & - & - & - & - & 1.4 & - & 0.3 & - & - & - & 3.1 & 1.0 & - & 99.8 \\
\hline $2-2,75-77$ & 11.9 & 41.9 & 2.3 & 0.9 & 2.3 & 26.0 & - & - & - & - & - & - & 17.9 & - & - & - & - & - & 7.8 & 1.0 & - & 100.1 \\
\hline $4-1,75-77$ & 29.3 & 73.1 & - & 1.0 & 1.7 & 16.9 & - & - & - & - & - & - & 4.0 & - & - & - & - & - & 3.0 & 0.3 & - & 100.0 \\
\hline $6-3,50$ & 51.25 & - & - & - & - & 0.6 & - & - & - & 87.1 & 5.2 & - & - & 1.0 & 4.8 & 1.3 & - & - & - & - & - & 100.0 \\
\hline $7-3,50$ & 60.80 & 8.5 & - & - & 0.4 & 1.1 & 0.4 & - & - & 11.3 & 14.8 & - & - & 0.4 & 53.7 & 9.5 & - & - & - & - & - & 100.1 \\
\hline $8-3,50$ & 70.3 & 65.6 & 1.7 & 1.0 & 3.8 & 23.1 & - & - & - & - & - & - & 0.3 & 0.3 & - & 0.3 & - & - & 2.4 & 1.4 & - & 99.9 \\
\hline $10-1,109-111$ & 86.7 & 70.0 & 2.4 & 1.7 & 1.4 & 19.2 & 0.3 & - & - & - & - & - & 1.4 & - & 0.7 & - & - & - & 1.0 & 1.5 & - & 99.6 \\
\hline $13-1,142-144$ & 107.0 & 66.4 & 0.7 & 2.0 & 2.0 & 26.5 & - & - & - & - & - & - & 0.7 & - & 0.3 & - & - & - & 1.3 & - & - & 99.9 \\
\hline $17-2,129-131$ & 183.5 & 74.9 & 1.4 & 1.4 & 0.4 & 14.8 & - & - & - & - & - & - & 0.7 & - & - & - & - & - & 5.8 & 04 & - & 99.8 \\
\hline $20-2,100-102$ & 240.2 & 11.3 & 28.6 & 3.0 & 2.0 & 3.6 & - & - & - & - & - & 0.3 & 45.8 & 2.6 & 0.7 & 1.0 & - & - & 0.7 & 0.3 & - & 99.9 \\
\hline $21-4,90-92$ & 262.3 & 63.8 & 1.8 & 2.1 & 2.1 & 19.5 & - & 0.4 & - & - & - & - & 2.5 & - & 2.1 & - & - & - & 5.3 & 0.4 & - & 100.0 \\
\hline $28-3,100-102$ & 393.9 & 2.5 & - & - & 0.4 & 1.8 & - & - & 14.4 & 3.2 & 33.8 & 6.5 & 1.8 & 1.4 & 24.1 & 9.5 & - & - & - & 0.7 & - & 100.1 \\
\hline $29-4,118-120$ & 405.1 & - & - & 0.7 & 0.7 & - & - & - & 1.0 & 0.3 & 56.5 & 4.1 & 7.5 & 0.3 & 19.0 & 9.5 & - & - & - & 0.3 & - & 99.8 \\
\hline $30-4,41-43$ & 413.7 & 2.6 & - & - & 0.6 & 0.3 & - & - & 0.3 & 6.1 & 80.5 & - & - & 0.6 & 2.2 & 6.1 & - & - & - & 0.6 & - & 99.9 \\
\hline $32-4,50-52$ & 432.9 & - & - & - & 0.3 & 0.6 & - & - & 1.0 & 7.5 & 28.4 & - & - & 1.3 & 1.3 & 59.5 & - & - & - & - & - & 99.9 \\
\hline Site 342 & & & & & & & & & & & & & & & & & & & & & & \\
\hline $1-2,49-51$ & 2.0 & 72.0 & 2.1 & 0.4 & 0.4 & 15.4 & - & - & 0.4 & - & - & - & 5.9 & - & 0.4 & - & - & - & 2.1 & 1.0 & - & 100.1 \\
\hline $1-5,69-71$ & 6.7 & 58.2 & 3.7 & 1.4 & 1.0 & 25.6 & - & - & - & - & - & 0.7 & 5.0 & - & - & - & - & & 4.0 & 0.3 & - & 99.9 \\
\hline $2 \cdot 2,94-96$ & 40.1 & 66.3 & 7.0 & 1.0 & 1.1 & 23.5 & - & - & - & - & - & - & - & - & 0.7 & - & - & - & - & 0.4 & - & 100.0 \\
\hline $3-4,100-102$ & 90.9 & - & 27.0 & - & 0.3 & - & - & - & 1.7 & 64.7 & - & - & - & 1.4 & 0.7 & 4.2 & - & - & - & - & - & 100.0 \\
\hline $4-2,110-112$ & 125.8 & 0.4 & 15.5 & - & 0.4 & 0.7 & - & - & 0.7 & 10.8 & - & - & - & 1.1 & 64.4 & 6.1 & - & - & - & - & - & 100.1 \\
\hline $100-102$ & 135.2 & - & 29.2 & - & - & - & - & - & - & 1.1 & - & - & - & 1.1 & 66.5 & 2.1 & - & - & - & - & - & 100.0 \\
\hline $5-6,100-102$ & 141.5 & - & 9.6 & - & - & - & - & - & - & 1.4 & - & - & - & 0.7 & 80.8 & 7.4 & - & - & - & - & - & 99.9 \\
\hline $6-4,61-62$ & 147.5 & - & 44.3 & - & 0.4 & 0.8 & - & - & - & 31.1 & - & - & - & 0.4 & 4.5 & 8.0 & - & - & - & 10.7 & - & 100.2 \\
\hline Site 343 & & & & & & & & & & & & & & & & & & & & & & \\
\hline $1-1,75-77$ & 0.2 & 48.4 & - & 0.4 & 0.7 & 13.8 & - & - & - & 2.2 & - & - & 32.0 & 0.4 & 0.4 & - & - & - & 0.7 & 1.1 & - & 100.1 \\
\hline $1-3,75-77$ & 1.2 & 50.0 & 7.1 & 0.7 & 0.3 & 11.8 & 1.4 & - & - & 0.3 & - & - & 25.3 & 0.6 & - & - & - & - & 1.4 & 1.1 & - & 100.0 \\
\hline $2-2,105-107$ & 5.7 & 58.2 & 6.6 & - & 0.4 & 25.6 & - & - & - & - & - & - & 1.1 & - & 0.4 & - & - & - & 1.1 & 6.7 & - & 100.2 \\
\hline $2-4,75-77$ & 8.7 & 53.3 & 10.1 & - & - & 24.3 & - & - & - & - & - & 0.4 & 9.8 & - & - & - & - & - & 0.4 & 1.8 & - & 100.2 \\
\hline $2, \mathrm{CC}$ & 12.5 & 63.3 & 10.2 & - & - & 17.8 & 0.4 & - & - & - & - & - & 6.9 & - & 0.4 & - & - & - & 0.4 & 0.7 & - & 100.1 \\
\hline $3-2,129-131$ & 53.5 & 72.7 & 7.5 & - & 0.6 & 15.9 & - & - & - & - & - & 0.3 & - & - & - & - & - & - & 2.6 & 0.3 & - & 99.9 \\
\hline $3-4,129-131$ & 56.0 & 38.6 & 20.8 & 14.0 & 14.7 & 10.9 & - & - & - & - & - & - & 0.3 & - & - & - & - & - & 0.7 & - & - & 100.0 \\
\hline $3-6,119-121$ & 59.7 & 83.9 & 0.3 & - & - & 12.3 & - & - & - & - & 0.3 & - & - & - & - & - & - & - & 2.3 & 0.7 & - & 99.8 \\
\hline $4-3,110-112$ & 102.6 & 55.2 & 0.7 & 0.3 & - & 11.7 & - & - & - & - & - & 0.3 & 26.9 & - & 0.3 & - & - & - & 3.4 & 1.0 & - & 99.8 \\
\hline $5-2,19-21$ & 147.3 & 51.6 & 17.0 & - & 0.4 & 28.9 & - & - & - & - & - & - & - & - & - & - & - & - & - & 2.1 & - & 100.0 \\
\hline $7-0,30-32$ & 202.0 & 1.0 & 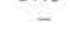 & - & 0.3 & 1.3 & - & 97.0 & - & - & - & - & - & - & 0.3 & - & - & - & - & - & - & 99.9 \\
\hline $8-3,100-103$ & 216.3 & 3.9 & - & 0.3 & 2.1 & 1.0 & - & 22.8 & - & - & - & - & 0.7 & - & 0.3 & 1.0 & - & 0.3 & 67.4 & - & - & 99.8 \\
\hline $11-2,130-132$ & 243.5 & 1.0 & - & 0.6 & 0.6 & 7.3 & - & 90.1 & - & 0.3 & - & - & - & - & - & - & - & - & - & - & - & 99.9 \\
\hline $15-1,99-101$ & 270.1 & 5.0 & - & 13.8 & 12.8 & 14.2 & - & 52.5 & - & - & - & - & 0.4 & - & 1.1 & - & - & 0.4 & - & - & - & 100.2 \\
\hline $16-3,18-20$ & 282.0 & 4.3 & - & - & 8.5 & 13.9 & - & 73.1 & - & - & - & - & - & - & - & - & - & - & - & 0.2 & - & 100.0 \\
\hline
\end{tabular}



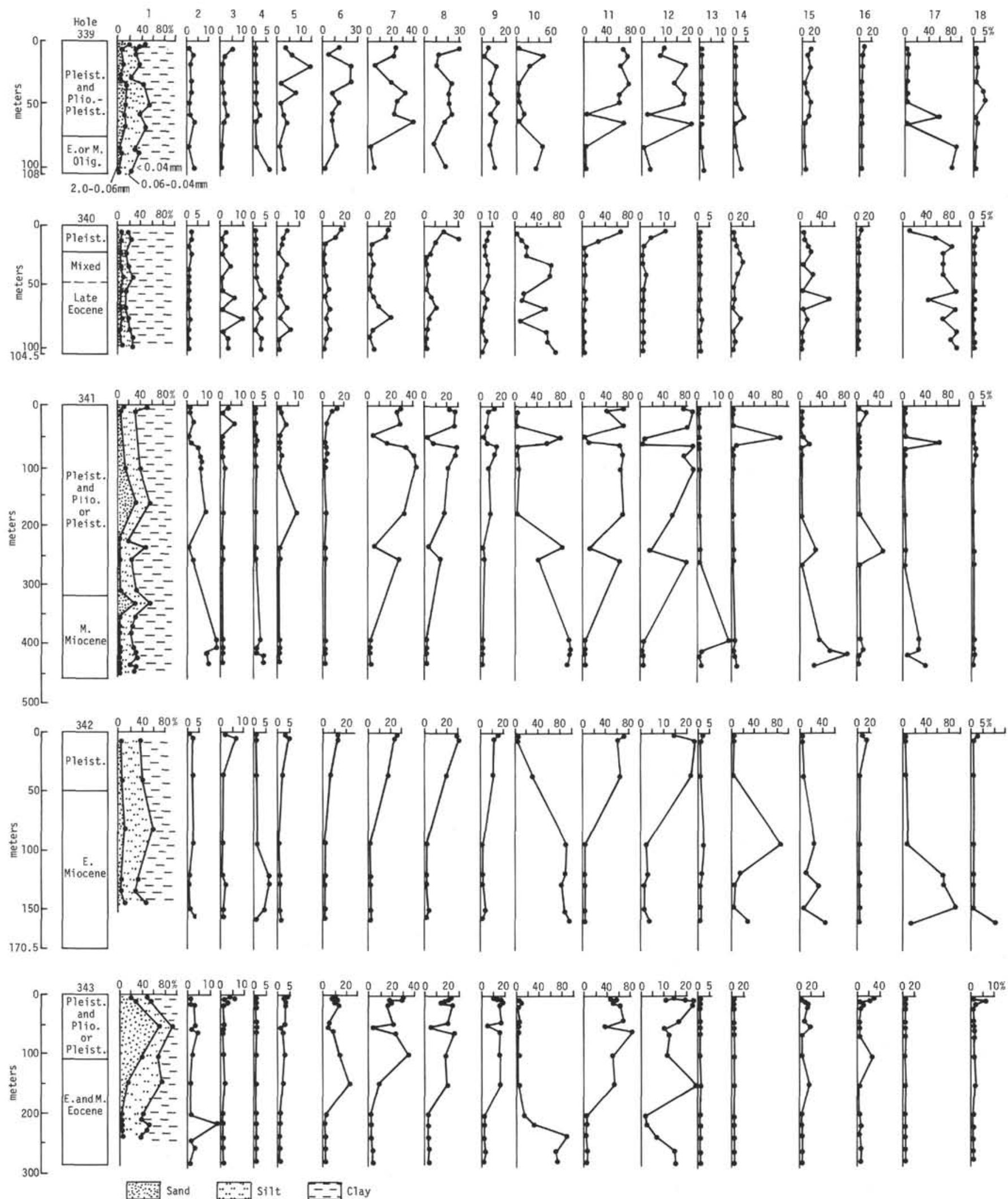

Figure 2. Lithologic diagrams of the sites. Legend: $1=$ grain size composition of sediments (\%), $2=$ content of heavy mineral fraction $0.1-0.5 \mathrm{~mm}(\%) ; 3-18=$ distribution of minerals, volcanic ash, biogenic material: $3=$ volcanic ash; $4=$ brown glass (basaltic); $5=$ clinopyroxene; $6=$ ilmenite $; 7=$ hornblende $; 8=$ epidote $; 9=$ garnet $; 10=F e$-sulfide $; 11=$ quartz; $12=$ feldspar; $13=$ light brown glass; 14 = colorless glass; $15=$ clayey aggregate; $16=$ biogenic carbonate; 17 = biogenic siliceous; and $18=$ glauconite. 
TABLE 3

Mineral Composition (\%) of Sediments Voring Plateau, DSDP Leg 38 X-ray Data ${ }^{a}$

\begin{tabular}{|c|c|c|c|c|c|c|c|c|c|c|c|}
\hline \multirow[b]{2}{*}{$\begin{array}{l}\text { Sample } \\
\text { (Interval } \\
\text { in } \mathrm{cm} \text { ) }\end{array}$} & \multirow[b]{2}{*}{$\begin{array}{c}\text { Depth } \\
\text { (m) }\end{array}$} & \multirow[b]{2}{*}{$\mathrm{CaCO}_{3}$} & \multicolumn{4}{|c|}{ Carbonates } & \multirow[b]{2}{*}{ స్ } & \multirow[b]{2}{*}{$\begin{array}{l}\text { 䔍 } \\
\tilde{\Xi}\end{array}$} & \multirow[b]{2}{*}{$\begin{array}{l}\frac{\mathscr{U}}{0} \\
\frac{\pi}{0} \\
\frac{0}{00} \\
\frac{\pi}{2}\end{array}$} & \multirow[b]{2}{*}{ 光䓴 } & \multirow[b]{2}{*}{ 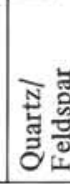 } \\
\hline & & & $\frac{\stackrel{\Xi}{0}}{\tilde{J}}$ & 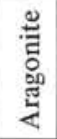 & 冚 & 总 & & & & & \\
\hline \multicolumn{12}{|l|}{ Site 339} \\
\hline $\begin{array}{l}2-2,140-142 \\
2-4,138-141 \\
3-1,129-131 \\
3-3,138-140 \\
3, \mathrm{CC} \\
4-1,142-144 \\
4-3,89-91 \\
4, \mathrm{CC} \\
5-2,138-140 \\
5, \mathrm{CC} \\
6-1,140-142 \\
6-4,85-87 \\
6, \mathrm{CC} \\
7-2,50-52 \\
8-5,60-62 \\
8, \mathrm{CC} \\
10, \mathrm{CC} \\
12-1,120-122\end{array}$ & $\begin{array}{l}10.9 \\
14.4 \\
18.8 \\
21.9 \\
27.0 \\
28.45 \\
31.0 \\
36.5 \\
39.5 \\
46.0 \\
47.4 \\
51.6 \\
55.5 \\
57.7 \\
72.0 \\
74.5 \\
93.5 \\
103.0\end{array}$ & $\begin{array}{r}12.51 \\
8.00 \\
6.00 \\
8.75 \\
2.50 \\
12.85 \\
12.76 \\
\overline{2} \\
2.50 \\
- \\
10.01 \\
2.50 \\
9.76 \\
3.76 \\
0.0 \\
0.0 \\
0.0 \\
0.0\end{array}$ & $\begin{array}{r}8 \\
6 \\
3 \\
7 \\
1 \\
10 \\
12 \\
0 \\
0 \\
2 \\
7 \\
0 \\
8 \\
0 \\
0 \\
0 \\
0 \\
0\end{array}$ & $\begin{array}{l}0 \\
+ \\
+ \\
+ \\
0 \\
0 \\
0 \\
0 \\
0 \\
0 \\
0 \\
0 \\
0 \\
0 \\
0 \\
0 \\
0 \\
0\end{array}$ & $\begin{array}{l}0 \\
+ \\
+ \\
+ \\
0 \\
+ \\
+ \\
0 \\
0 \\
0 \\
0 \\
+ \\
+ \\
0 \\
0 \\
0 \\
0 \\
0\end{array}$ & $\begin{array}{l}0 \\
0 \\
0 \\
0 \\
0 \\
0 \\
0 \\
0 \\
0 \\
0 \\
0 \\
0 \\
0 \\
0 \\
0 \\
0 \\
0 \\
0\end{array}$ & $\begin{array}{c}8 \\
- \\
- \\
- \\
1 \\
- \\
- \\
0 \\
0 \\
2 \\
7 \\
- \\
- \\
0 \\
0 \\
0 \\
0 \\
0\end{array}$ & $\begin{array}{r}13 \\
14 \\
12 \\
15 \\
19 \\
11 \\
19 \\
14 \\
8 \\
19 \\
24 \\
15 \\
12 \\
10 \\
12 \\
20 \\
0 \\
0\end{array}$ & $\begin{array}{r}3 \\
2 \\
2 \\
3 \\
10 \\
3 \\
3 \\
3 \\
2 \\
2 \\
2 \\
3 \\
3 \\
2 \\
3 \\
4 \\
0 \\
0\end{array}$ & $\begin{array}{l}1 \\
1 \\
2 \\
1 \\
4 \\
1 \\
3 \\
1 \\
+ \\
1 \\
1 \\
4 \\
+ \\
1 \\
1 \\
1 \\
2 \\
1 \\
0 \\
0\end{array}$ & $\begin{array}{l}3.3 \\
4.7 \\
3.0 \\
3.8 \\
1.4 \\
2.8 \\
3.2 \\
3.5 \\
- \\
6.3 \\
4.0 \\
- \\
3.0 \\
3.3 \\
2.4 \\
4.0 \\
- \\
-\end{array}$ \\
\hline \multicolumn{12}{|l|}{ Site 340} \\
\hline $\begin{array}{l}1-1,75-77 \\
1-2,148-150 \\
1-5,75-77 \\
1, \text { CC } \\
2-2,75-77 \\
2-3,80-82 \\
2, \text { CC } \\
3-1,75-77 \\
3-2,75-77 \\
3-4,75-77 \\
3, \text { CC } \\
4-3,75-77 \\
4, \text { CC } \\
6, \text { CC } \\
7-4,95-97 \\
8-1,72-74 \\
8-5,72-74 \\
9-1,70-72 \\
9, \text { CC } \\
10-3,50-52 \\
10-5,50-52 \\
11-3,81-83 \\
11, \text { CC }\end{array}$ & $\begin{array}{r}0.8 \\
3.2 \\
7.2 \\
9.5 \\
11.9 \\
13.5 \\
19.0 \\
19.8 \\
21.4 \\
24.6 \\
28.5 \\
33.5 \\
38.0 \\
57.0 \\
62.8 \\
67.2 \\
73.6 \\
76.7 \\
85.5 \\
89.2 \\
92.4 \\
99.0 \\
104.5\end{array}$ & $\begin{array}{l}- \\
- \\
- \\
\overline{-} \\
\overline{-} \\
\overline{0} .0 \\
- \\
\overline{1} \\
1.50 \\
2.0 \\
0.0 \\
0.0 \\
0.0 \\
0.0 \\
0.0 \\
- \\
\overline{0} \\
0.0 \\
0.0 \\
0.0 \\
0.0 \\
3.0\end{array}$ & $\begin{array}{l}3 \\
7 \\
4 \\
7 \\
0 \\
4 \\
2 \\
2 \\
0 \\
8 \\
1 \\
0 \\
1 \\
0 \\
0 \\
0 \\
0 \\
0 \\
0 \\
0 \\
0 \\
0 \\
0\end{array}$ & $\begin{array}{l}0 \\
0 \\
0 \\
0 \\
0 \\
0 \\
0 \\
0 \\
0 \\
0 \\
0 \\
0 \\
0 \\
0 \\
0 \\
0 \\
0 \\
0 \\
0 \\
0 \\
0 \\
0 \\
0\end{array}$ & $\begin{array}{l}0 \\
+ \\
0 \\
0 \\
0 \\
0 \\
0 \\
0 \\
0 \\
0 \\
0 \\
0 \\
0 \\
0 \\
0 \\
0 \\
0 \\
0 \\
0 \\
0 \\
0 \\
0 \\
0\end{array}$ & $\begin{array}{l}0 \\
0 \\
0 \\
0 \\
0 \\
0 \\
0 \\
0 \\
0 \\
0 \\
0 \\
0 \\
0 \\
0 \\
0 \\
0 \\
0 \\
0 \\
0 \\
0 \\
0 \\
0 \\
0\end{array}$ & $\begin{array}{l}3 \\
- \\
4 \\
7 \\
0 \\
4 \\
2 \\
2 \\
0 \\
8 \\
1 \\
0 \\
1 \\
0 \\
0 \\
0 \\
0 \\
0 \\
0 \\
0 \\
0 \\
0 \\
0\end{array}$ & $\begin{array}{r}10 \\
14 \\
8 \\
16 \\
2 \\
12 \\
22 \\
7 \\
1 \\
7 \\
3 \\
1 \\
4 \\
3 \\
2 \\
1 \\
1 \\
1 \\
1 \\
2 \\
1 \\
1 \\
2\end{array}$ & $\begin{array}{l}2 \\
4 \\
3 \\
4 \\
+ \\
5 \\
5 \\
8 \\
6 \\
0 \\
6 \\
2 \\
0 \\
2 \\
4 \\
2 \\
+ \\
+ \\
+ \\
+ \\
+ \\
+ \\
2 \\
2\end{array}$ & $\begin{array}{l}+ \\
3 \\
+ \\
2 \\
0 \\
1 \\
2 \\
2 \\
0 \\
2 \\
+ \\
+ \\
0 \\
+ \\
1 \\
0 \\
0 \\
0 \\
0 \\
+ \\
0 \\
0 \\
0 \\
0\end{array}$ & $\begin{array}{c}- \\
2.0 \\
- \\
2.7 \\
- \\
2.0 \\
2.2 \\
0.9 \\
- \\
0.9 \\
- \\
- \\
- \\
0.6 \\
1.0 \\
- \\
- \\
- \\
- \\
- \\
1.0 \\
0.5 \\
1.0\end{array}$ \\
\hline \multicolumn{12}{|l|}{ Site 341} \\
\hline $\begin{array}{l}7, \mathrm{CC} \\
8, \mathrm{CC} \\
9, \mathrm{CC} \\
10-3,109-111 \\
10, \mathrm{CC} \\
11-3,141-143 \\
11, \mathrm{CC} \\
12-3,135-137 \\
12, \mathrm{CC} \\
13, \mathrm{CC} \\
14, \mathrm{CC} \\
15, \mathrm{CC} \\
16, \mathrm{CC} \\
17, \mathrm{CC} \\
18, \mathrm{CC} \\
19, \mathrm{CC} \\
20, \mathrm{CC}\end{array}$ & $\begin{array}{r}66.5 \\
76.0 \\
85.5 \\
90.4 \\
95.0 \\
99.7 \\
104.5 \\
105.0 \\
105.5 \\
114.0 \\
123.5 \\
161.0 \\
171.0 \\
190.0 \\
209.0 \\
228.0 \\
247.0\end{array}$ & $\begin{array}{r}5.50 \\
4.00 \\
8.26 \\
8.50 \\
- \\
\overline{9} \\
9.00 \\
\overline{-} \\
9.51 \\
10.76 \\
15.76 \\
\overline{8} \\
8.00 \\
\overline{8} \\
8.76 \\
9.00 \\
10.51\end{array}$ & $\begin{array}{r}2 \\
4 \\
8 \\
7 \\
6 \\
4 \\
6 \\
10 \\
6 \\
8 \\
14 \\
8 \\
6 \\
5 \\
7 \\
10 \\
7\end{array}$ & $\begin{array}{l}0 \\
0 \\
0 \\
0 \\
0 \\
0 \\
0 \\
0 \\
0 \\
0 \\
0 \\
0 \\
0 \\
0 \\
0 \\
0 \\
0\end{array}$ & $\begin{array}{l}0 \\
0 \\
0 \\
0 \\
0 \\
0 \\
0 \\
0 \\
0 \\
0 \\
0 \\
0 \\
0 \\
0 \\
0 \\
0 \\
0\end{array}$ & $\begin{array}{l}0 \\
0 \\
0 \\
0 \\
0 \\
0 \\
0 \\
0 \\
0 \\
0 \\
0 \\
0 \\
0 \\
0 \\
0 \\
0 \\
0\end{array}$ & $\begin{array}{r}2 \\
4 \\
8 \\
7 \\
6 \\
4 \\
6 \\
10 \\
6 \\
8 \\
14 \\
8 \\
6 \\
5 \\
7 \\
10 \\
7\end{array}$ & $\begin{array}{l}11 \\
18 \\
13 \\
12 \\
16 \\
16 \\
17 \\
19 \\
14 \\
22 \\
17 \\
25 \\
17 \\
17 \\
13 \\
20 \\
14\end{array}$ & $\begin{array}{r}6 \\
7 \\
7 \\
7 \\
6 \\
7 \\
6 \\
7 \\
5 \\
6 \\
5 \\
11 \\
7 \\
6 \\
5 \\
6 \\
6\end{array}$ & $\begin{array}{l}2 \\
2 \\
2 \\
2 \\
2 \\
2 \\
+ \\
2 \\
2 \\
2 \\
2 \\
1 \\
1 \\
4 \\
2 \\
2 \\
1 \\
2 \\
2\end{array}$ & $\begin{array}{l}1.4 \\
2.0 \\
1.4 \\
1.3 \\
2.0 \\
- \\
2.0 \\
2.1 \\
2.0 \\
2.8 \\
2.8 \\
1.7 \\
1.9 \\
2.1 \\
2.2 \\
2.5 \\
1.8\end{array}$ \\
\hline
\end{tabular}


TABLE 3 - Continued

\begin{tabular}{|c|c|c|c|c|c|c|c|c|c|c|c|}
\hline \multirow[b]{2}{*}{$\begin{array}{c}\text { Sample } \\
\text { (Interval } \\
\text { in } \mathrm{cm} \text { ) }\end{array}$} & \multirow[b]{2}{*}{$\begin{array}{c}\text { Depth } \\
\text { (m) }\end{array}$} & \multirow[b]{2}{*}{$\mathrm{CaCO}_{3}$} & \multicolumn{4}{|c|}{ Carbonates } & \multirow[b]{2}{*}{ 푱 } & \multirow[b]{2}{*}{ 营 } & \multirow[b]{2}{*}{$\begin{array}{l}\frac{8}{0} \\
\frac{\pi}{0} \\
\frac{0}{60} \\
\frac{\pi}{2}\end{array}$} & \multirow[b]{2}{*}{ 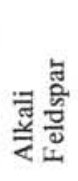 } & \multirow[b]{2}{*}{ 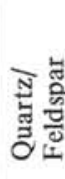 } \\
\hline & & & 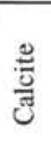 & 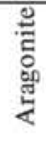 & 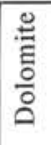 & 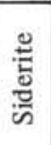 & & & & & \\
\hline $21-2,120-122$ & 259.4 & - & 3 & 0 & 0 & 0 & 3 & 13 & 5 & 2 & 1.9 \\
\hline $21, \mathrm{CC}$ & 266.0 & 0.25 & 0 & 0 & 0 & 0 & 0 & 22 & 6 & 2 & 2.8 \\
\hline $23, \mathrm{CC}$ & 313.5 & - & 0 & 0 & 0 & 0 & 0 & 14 & 5 & 2 & 2.0 \\
\hline $23-3,10-12$ & 304.1 & 3.00 & 2 & 0 & 0 & 0 & 2 & 12 & 5 & + & - \\
\hline $24-3,75-77$ & 317.5 & - & 4 & 0 & 0 & 0 & 4 & 8 & 4 & 1 & 1.6 \\
\hline $24, \mathrm{CC}$ & 323.0 & 2.50 & 0 & 0 & 0 & 0 & 0 & 14 & 4 & 1 & 2.8 \\
\hline $25-3,75-77$ & 339.0 & - & 0 & 0 & 0 & 0 & 0 & 4 & + & + & - \\
\hline $25, \mathrm{CC}$ & 342.0 & 0.25 & 0 & 0 & 0 & 0 & 0 & 10 & + & + & - \\
\hline $26-5,137-139$ & 359.2 & - & 6 & 0 & 0 & 0 & 6 & 3 & + & + & - \\
\hline $26, \mathrm{CC}$ & 361.0 & 10.0 & 8 & 0 & 0 & 0 & 8 & 4 & 1 & 0 & 4.0 \\
\hline $27-4,120-122$ & 376.6 & - & 7 & 0 & 0 & 0 & 7 & 3 & 0 & 0 & - \\
\hline $28, \mathrm{CC}$ & 399.0 & 0.50 & 0 & 0 & 0 & 0 & 0 & 4 & 0 & 0 & - \\
\hline $31-4,28-30$ & 423.1 & - & 0 & 0 & 0 & 0 & 0 & 7 & + & + & - \\
\hline $33-4,60-62$ & 442.4 & - & 0 & 0 & 0 & 0 & 0 & 3 & 0 & 0 & - \\
\hline $34, \mathrm{CC}$ & 456.0 & 0.25 & 0 & 0 & 0 & 0 & 0 & 3 & 0 & 0 & - \\
\hline \multicolumn{12}{|l|}{ Site 342} \\
\hline $1-1,49-51$ & 0.5 & 11.26 & 6 & 0 & + & 0 & - & 18 & 2 & 1 & 6.0 \\
\hline $1-4,49-51$ & 5.0 & - & 7 & 0 & + & 0 & _- & 10 & 2 & 1 & 3.3 \\
\hline $1-6,69-71$ & 8.2 & - & + & 0 & 0 & 0 & + & 40 & 6 & 2 & 5.0 \\
\hline $1, \mathrm{CC}$ & 9.0 & 6.50 & 4 & 0 & 0 & 0 & 4 & 17 & 5 & 2 & 2.4 \\
\hline $2-1,99-101$ & 38.6 & - & 4 & 3 & 0 & 0 & 7 & 11 & 4 & 1 & 2.2 \\
\hline $2-4,80-82$ & 43.1 & - & 0 & 6 & 0 & 0 & 6 & 16 & 5 & 2 & 2.3 \\
\hline $2, \mathrm{CC}$ & 47.60 & 14.76 & 10 & 0 & 0 & 0 & 10 & 12 & 4 & 1 & 4.0 \\
\hline $3-2,110-112$ & 87.8 & - & 0 & 0 & 0 & 0 & 0 & 6 & 1 & 0 & 6.0 \\
\hline $3-3,119-121$ & 89.6 & - & 0 & 0 & 0 & 0 & 0 & 5 & 1 & 0 & 5.0 \\
\hline $4-1,110-112$ & 124.2 & - & 0 & 0 & 0 & 0 & 0 & 3 & + & + & - \\
\hline $5, \mathrm{CC}$ & 142.0 & - & 0 & 0 & 0 & 0 & 0 & 2 & 0 & 0 & - \\
\hline $6-3,90-92$ & 146.2 & - & 0 & 0 & 0 & 0 & 0 & 3 & 0 & 0 & - \\
\hline $6, \mathrm{CC}$ & 151.5 & - & 0 & 0 & 0 & 0 & 0 & 2 & + & 0 & - \\
\hline
\end{tabular}

Site 343

\begin{tabular}{lrlrllllrrrr}
$2-1,75-77$ & 3.9 & - & 2 & 0 & 0 & 0 & 2 & 16 & 6 & 2 & 2.0 \\
$3-1,129-131$ & 51.8 & - & 3 & 0 & 0 & 0 & 3 & 27 & 10 & 4 & 1.9 \\
$3-5,120-122$ & 57.4 & - & 2 & 0 & 0 & 0 & 2 & 20 & 7 & 3 & 2.0 \\
$3, \mathrm{CC}$ & 60.0 & - & 2 & 0 & 0 & 0 & 2 & 40 & 6 & 2 & 5.0 \\
$4-1,119-121$ & 99.3 & - & 0 & 0 & + & 0 & + & 12 & 5 & 2 & 1.7 \\
$4-4,119-121$ & 104.0 & - & 0 & 0 & 0 & 0 & 0 & 16 & 4 & 2 & 2.0 \\
$4, \mathrm{CC}$ & 107.5 & - & 2 & 0 & 0 & 0 & 2 & 18 & 5 & 2 & 2.5 \\
$5-4,114-116$ & 151.5 & - & 0 & 0 & 0 & 0 & 0 & + & + & + & - \\
$6, \mathrm{CC}$ & 202.5 & - & 0 & 0 & 0 & 0 & 0 & 12 & + & + & - \\
$7-2,90-92$ & 205.6 & - & 0 & 0 & 0 & 0 & 0 & 5 & 0 & + & - \\
$7-3,89-91$ & 206.6 & - & 0 & 0 & 0 & 0 & 0 & 11 & + & + & - \\
$7, \mathrm{CC}$ & 212.0 & - & 0 & 0 & 0 & + & + & 11 & 0 & + & - \\
$8-1,100-102$ & 213.1 & - & 0 & 0 & 0 & 0 & 0 & 12 & + & + & - \\
$8-2,50-52$ & 214.1 & - & 0 & 0 & 0 & 0 & 0 & 8 & + & + & - \\
$8, \mathrm{CC}$ & 221.5 & - & 0 & 0 & 0 & 0 & 0 & 8 & + & + & - \\
$9-2,110-112$ & 224.2 & - & 0 & 0 & 0 & 0 & 0 & 14 & 2 & 1 & 4.6 \\
$10-1,80-82$ & 231.9 & - & 0 & 0 & 0 & 0 & 0 & 7 & + & + & - \\
$11-1,118-120$ & 241.8 & - & 0 & 0 & 0 & 0 & 0 & 11 & + & + & - \\
$16-1,119-121$ & 279.7 & - & 4 & 0 & 0 & 0 & 4 & 9 & + & 1 & - \\
$16-2,110-112$ & 281.0 & - & 0 & 0 & 0 & 0 & 0 & 3 & + & + & - \\
\hline
\end{tabular}

${ }^{\mathrm{a}}$ Analytical procedure of L.N. Yorbunova (1969) and Eltzina (1973); $0=$ absent, $+=$ present,$-=$ not determined.

the basement of glacial strata, aragonite is present $(3 \%$ $6 \%$ ), and may represent relicts of pteropod fauna. Petrographic data indicate the calcite consists of coccoliths, sometimes foraminifera. It is possible that the peaks of increased calcite content in the Pleistocene deposits correspond to interglacial periods. where the conditions for nannoplankton development were improved and the influx of terrigenous material was decreased.

Generally, carbonates are absent in Pliocene-Oligocene sediments. The small concentrations increase only in late Miocent sediments (up to 8\%, Site 342). Eocene 
sediments are also noncalcareous. Only in the basement, Eocene sediments at Site 348 are carbonates present $(4 \%)$.

\section{Quartz}

High (20\%-25\%, up to $40 \%)$ contents of quartz are characteristic for Plio-Pleistocene deposits. The maximum content corresponds to periods of maximum icerafting. This is indicated by a reversed correlation between the quartz and calcite distributions in the majority of samples (Figure 3). The lower parts of PlioPleistocene strata, with the interbeds of underlying diatom ooze, are appreciably impoverished in quartz. The quartz content in Pliocene and Miocene sediments is not very large, up to $5 \%-10 \%$ in Pliocene and late Miocene, and only up to $1 \%-3 \%$ in older strata. Diatom ooze of Oligocene and late and middle Eocene age contains very little quartz; however, in early Eocene (Site 343) the terrigenous component increases, and the quartz component reaches up to $10 \%-14 \%$.

\section{Feldspars}

Almost without exception, plagioclases are abundant among the feldspars. In Plio-Pleistocene sediments, their content is up to $10 \%-11 \%$; orthoclases-not more than $3 \%-4 \%$. The correlation between quartz and feldspars (Figure 3 ) in the Pleistocene is evidence of their similar derivation.

The feldspar content decreases in Pliocene-Eocene sediments. This is also characteristic for quartz; however, a correlation between these two minerals is not present. Plagioclase in Tertiary diatom oozes are of
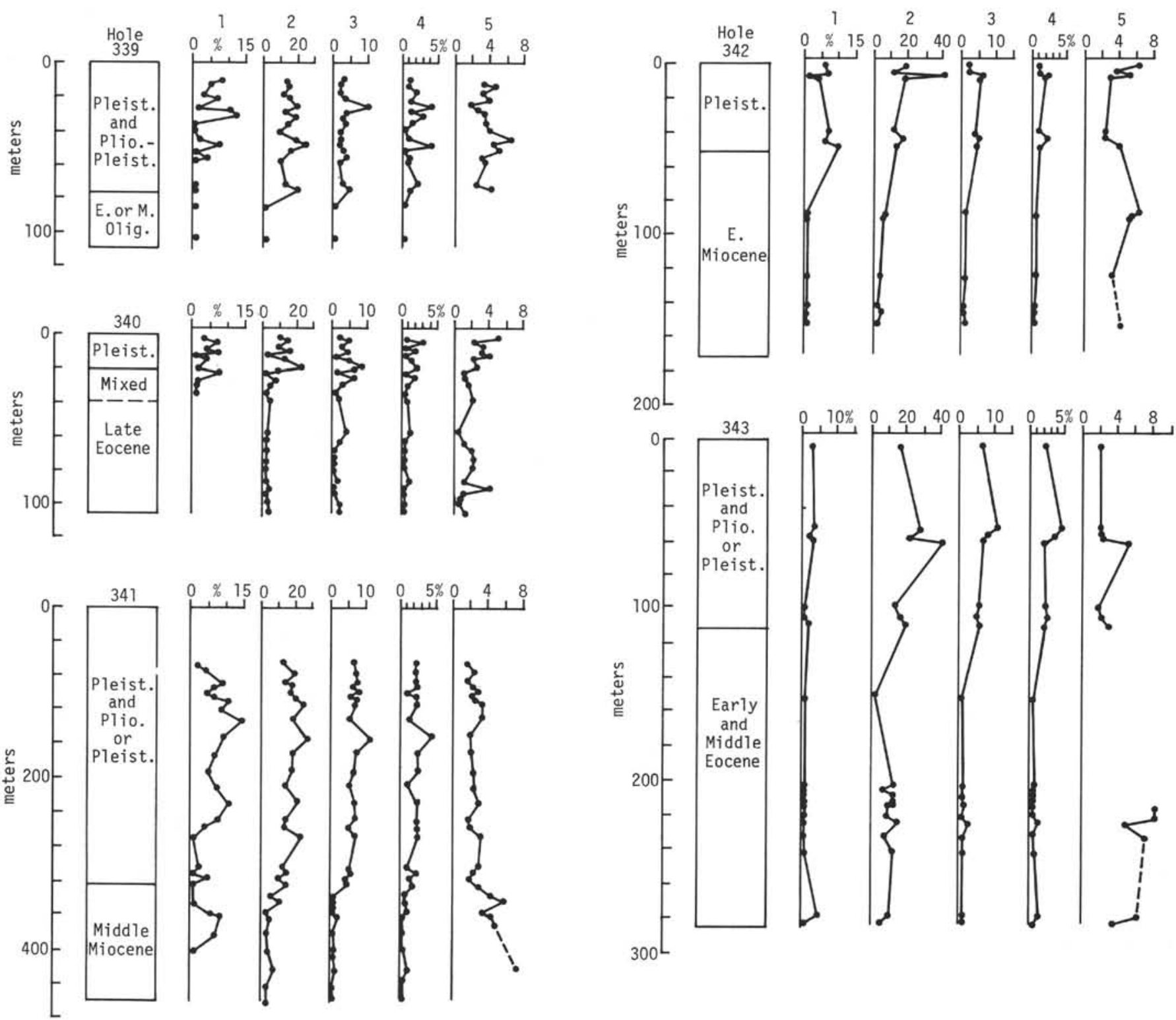

Figure 3. Distribution of minerals in sediment samples of the Vbring Plateau (\%). Legend: $1=$ carbonates; $2=$ quartz; $3=$ plagioclase; $4=K$-feldspar; 5 = quartz-feldspar $(Q / F)$ ratio. 
volcanic origin, as shown by the presence of volcanic ash and glass in silty fractions (Figure 2). In the early Eocene terrigenous strata where turbidite interbeds are also present, feldspars have a direct correlation with quartz.

\section{Quartz to Feldspar Ratios (Q/F)}

High ratios (up to 6-8) are characteristic of PlioPleistocene deposits. This may be a result of the prevalence of erosional products from quartzites and sandstones, as well as an influx of weathering products from ancient preglacial rocks. In the Pliocene (Site 342) and the late and middle Miocene, the $\mathrm{Q} / \mathrm{F}$ ratio is also high.

In Eocene siliceous sediments, the $\mathrm{Q} / \mathrm{F}$ ratio is often equal to 1 or $<1$, an indication of a volcanogenic source for the plagioclases (Site 340). In early Eocene terrigenous sediments (Site 343), the ratio increases.

\section{CHEMICAL COMPOSITION OF SEDIMENTS}

Sediments from Sites 339, 340, 341, 342 differ considerably in their chemical composition (Table 4). The Pleistocene terrigenous glacial-marine sediments contain increased amounts of $\mathrm{CaCO}_{3}$ and $\mathrm{K}_{2} \mathrm{O}$. $\mathrm{C}_{\alpha \mathbf{g}}$ content is less than in Recent terrigenous sediments of the Norwegian Sea (Emelyanov et al., 1975). For other elements, the glacial-marine sediments are similar to the composition of granitoids of the Baltic shield and Quaternary moraines (Table 5). There is no doubt that the sediments, as well as moraines, were formed mainly by the products of erosion of the Baltic crystalline shield.

Pleistocene terrigenous muds from Site 342 have a similar composition with the exception of a higher content of $\mathrm{Fe}, \mathrm{Mn}$, and $\mathrm{Ti}$. This indicates a similarity with the typical deep-sea terrigenous muds of the Atlantic Ocean (Emelyanov et al., 1975).

Late Miocene diatomites differ from the diatom oozes of Site 340 by the following: (1) higher contents of $\mathrm{CaCO}_{3}$ and $\mathrm{CO}_{\text {org }} ;(2)$ higher contents of $\mathrm{Fe}, \mathrm{Ti}, \mathrm{Ni}$, $\mathrm{Cr}$, and $\mathrm{K}_{2} \mathrm{O}$, and (3) a low content of $\mathrm{Na}_{2} \mathrm{O}$. Miocene diatom mudstones from Site 341 and the early Pleistocene of Site 342 are characterized by similar chemical compositions. However, in contrast to the diatomites, high $\mathrm{C}_{\text {org }}$ contents (up to $2.52 \%$ ) are present. This is the maximum value for the sediments from all sites of the $\mathrm{V} \phi$ ring Plateau. The contents of $\mathrm{Fe}$ (up to $6.00 \%$ ), $\mathrm{Ti}$ (up to $0.63 \%$ ), $\mathrm{Cr}$ (up to $10.30 .10^{-4 \%}$ ) are noticeable.

Volcanogenic sediments of the Norwegian Sea are characterized by high amounts of $\mathrm{Fe}, \mathrm{Ti}, \mathrm{Ni}, \mathrm{Cr}$, and $\mathrm{K}_{2} \mathrm{O}$. This is indicative of the close relationship of the diatomites and diatom sediments of Sites 341 and 342 with volcanic processes (Emelyanov and Kharin, 1974; Emelyanov et al., 1975). Also noted is the presence of volcanic glass in the Eocene sediments (Figure 2).

Eocene oozes (Site 340) are contrasted to glacialmarine and terrigenous sediments by the following features: (1) low $\mathrm{CaCO}_{3}$ content, (2) low $\mathrm{C}_{\text {org }}, \mathrm{Fe}, \mathrm{Mn}$, $\mathrm{Ti}, \mathrm{P}, \mathrm{Cr}$, and $\mathrm{K}_{2} \mathrm{O}$ content, and (3) a high $\mathrm{Na}_{2} \mathrm{O}$ content. These chemical characteristics make the diatom oozes of the V $\phi$ ring Plateau similar to the Recent diatom oozes of the deep-sea Antarctic zone of the Atlantic Ocean (Emelyanov et al., 1975). Radiolaria and diatom-spicule oozes have a chemical composition similar to the diatom oozes.

\section{DISCUSSION AND CONCLUSIONS}

Based on the mineral and chemical data, the origin and depositional conditions of the sedimentary deposits can be considered.

Thick deposits of glacial-marine sediments accumulated during the Pleistocene on the V $\phi$ ring Plateau, especially in the region of Sites 341 and 343 . The most active sedimentation probably took place during early periods of glaciation, when the weathered materials of ancient rocks were glaciated. These materials, represented mainly by quartz and clay minerals, formed the glacial-marine sediments of the V $\phi$ ring Plateau. Upper horizons of glacial-marine sediments contain appreciable quantities of biogenous carbonates.

Terrigenous material represents glaciated crystalline rocks. Since feldspars are slightly destroyed under such conditions, the $\mathrm{Q} / \mathrm{F}$ ratio in the upper deposits of glacial sediments is lower in comparison with the underlying strata. Thus, mineralogical data indicate the relationship of the glacial sediments with the rocks of the contiguous land with crystalline rocks (granites), and Cambrian-Silurian calcareous rocks (northern Norway) which contributed sediments. The latter source is indicated by the admixture of dolomite in the sediments of Site 339. The mineral compositions of Quaternary and Recent sediments of the eastern region of the Norwegian Sea are nearly identical (Holtedahl, 1955; Emelyanov et al., 1975).

The chemical composition of the terrigenous sediments has a close relationship with the average composition of glacially eroded acid rocks, granitoids of the Baltic shield, and Quaternary moraines. The transportation of minerals by floating ice and icebergs did not provide a thick accumulation of Pleistocene sediments on the V $\phi$ ring Plateau. It is a safe assumption that the main part of products of glacial origin was provided during the time of glacial erosion. Glaciers, during the maximum of glaciation, sank to the 200meter isobath (Holtedahl, 1957, 1958). After deglaciation, the shelf moraines were submerged and subjected to marine erosion. The transportation of minerals was provided by ice, icebergs, currents, and on the slopes of the V $\phi$ ring Plateau, by suspension currents.

Marine sedimentation prevailed in the development of the V $\phi$ ring Plateau. The sediments are characterized by the presence of glaciated rocks, admixtures of the products of moraines, and fragments from underlying deposits. Greenland, Iceland, and Jan-Mayen volcanic contributions were minor, as shown by the absence of volcanic ash and the chemical composition of the sediments.

Other types of sedimentation took place before the Quaternary and early Pleistocene (Sites 339 and 340). Biogenic siliceous oozes became prevalent. Therefore, before the Quaternary a different hydrochemical and tectonic regime existed in the Norwegian Sea. This 
TABLE 4

Chemical Composition of Sediments from V $\phi$ ring Plateau, DSDP Leg 38

\begin{tabular}{|c|c|c|c|c|c|c|c|c|c|c|c|c|c|c|c|c|}
\hline \multirow{2}{*}{$\begin{array}{c}\text { Sample } \\
\text { (Interval } \\
\text { in } \mathrm{cm} \text { ) }\end{array}$} & \multirow{2}{*}{$\begin{array}{l}\text { Depth } \\
\text { (m) }\end{array}$} & \multicolumn{6}{|c|}{ Content $(\%)$} & \multicolumn{6}{|c|}{ Content $\left(\%-10^{-4}\right)$} & \multicolumn{2}{|c|}{ Content $(\%)$} & \multirow{2}{*}{$\begin{array}{c}\text { Sedimen } \\
\text { Type }^{\mathrm{a}}\end{array}$} \\
\hline & & $\mathrm{CaCO}_{3}$ & $\mathrm{C}_{\text {org }}$ & $\mathrm{Fe}$ & $\mathrm{Mn}$ & $\mathrm{Ti}$ & $P$ & $\mathrm{Cu}$ & Ln & $\mathrm{Ni}$ & Co & $\mathrm{Cr}$ & $\mathrm{Cd}$ & $\mathrm{K}_{2} \mathrm{O}$ & $\mathrm{Na}_{2} \mathrm{O}$ & \\
\hline \multicolumn{17}{|l|}{ Site 339} \\
\hline $2-2,119-121$ & 10.8 & 13.01 & 0.60 & 4.40 & 0.05 & 0.54 & 0.07 & 32 & 85 & 44 & 10 & 84 & $<4$ & & 2.43 & M-gl.m. \\
\hline $2-2,140-142$ & 10.9 & 12.51 & 0.45 & 3.96 & 0.05 & 0.56 & 0.6 & 23 & 79 & 38 & 10 & 84 & $<4$ & 3.15 & 2.00 & M-gl.m. \\
\hline $2-4,138-141$ & 14.4 & 8.00 & 0.30 & 3.62 & 0.05 & 0.52 & 0.06 & 32 & 79 & 49 & 15 & 79 & $<4$ & 3.06 & 1.98 & M-gl.m. \\
\hline $3-2,110-112$ & 20.2 & 6.00 & 0.45 & 3.86 & 0.04 & 0.52 & 0.07 & 25 & 87 & 44 & 12 & 74 & $<4$ & 2.96 & 2.18 & M-gl.m. \\
\hline $3-3,138-140$ & 21.9 & 8.75 & 0.39 & 4.34 & 0.05 & 0.54 & 0.07 & 35 & 92 & 46 & 18 & 86 & $<4$ & 2.90 & 2.21 & M-gl.m. \\
\hline $3, \mathrm{CC}$ & 27.0 & 2.50 & 0.34 & 3.80 & 0.03 & 0.54 & 0.06 & 33 & 87 & 27 & 27 & 87 & $<6$ & 2.72 & 2.04 & M-gl.m. \\
\hline $4-1,142-144$ & 28.4 & 12.85 & 0.60 & 4.27 & 0.08 & 0.56 & 0.06 & 35 & 100 & 49 & 22 & 101 & $<6$ & 2.87 & 2.10 & M-gl.m. \\
\hline $4-3,89-91$ & 31.0 & 12.76 & 0.63 & 4.27 & 0.06 & 0.50 & 0.06 & 32 & 86 & 50 & 21 & 105 & $<6$ & 3.11 & 1.72 & M-gl.m. \\
\hline $4-4,17-19$ & 32.0 & 13.51 & 0.81 & 4.05 & 0.06 & 0.50 & 0.06 & 25 & 80 & 45 & 22 & 105 & $<6$ & 3.82 & 1.67 & M-gl.m. \\
\hline $5-2,138-140$ & 39.5 & 2.50 & 0.60 & 3.70 & 0.05 & 0.47 & 0.04 & 24 & 67 & 22 & 18 & 78 & $<6$ & 2.40 & 1.92 & M-gl.m. \\
\hline $6-2,23-25$ & 47.9 & $10: 01$ & 0.42 & 3.55 & 0.02 & 0.43 & 0.04 & 27 & 85 & 30 & 15 & 80 & $<6$ & 2.30 & 1.77 & M-gl.m. \\
\hline $6-4,85-87$ & 51.6 & 2.50 & 0.54 & 3.86 & 0.06 & 0.48 & 0.05 & 27 & 78 & 27 & 17 & 83 & $<6$ & 2.28 & 1.85 & M-gl.m. \\
\hline $6, \mathrm{CC}$ & 55.5 & 9.76 & 0.75 & 3.80 & 0.05 & 0.47 & 0.05 & 23 & 89 & 42 & 19 & 97 & $<6$ & 2.49 & 1.93 & M-gl.m. \\
\hline $7-2,50-52$ & 57.5 & 3.76 & 0.45 & 3.10 & 0.03 & 0.30 & 0.03 & 40 & 83 & 40 & 15 & 47 & $<6$ & 1.81 & 3.67 & T-si. \\
\hline $7-3,50-52$ & 59.2 & 6.25 & 0.39 & 3.19 & 0.04 & 0.30 & 0.03 & 40 & 82 & 30 & 15 & 49 & $<6$ & 1.84 & 3.50 & T-si. \\
\hline $7, \mathrm{CC}$ & 65.0 & 2.50 & 0.54 & 2.08 & 0.03 & 0.22 & 0.02 & 26 & 54 & 29 & 17 & 41 & $<6$ & 1.64 & 2.72 & D. \\
\hline $8-4,10-12$ & 69.9 & 0.00 & 0.51 & 3.80 & 0.06 & 0.50 & 0.05 & 25 & 85 & 36 & 22 & 83 & $<6$ & 2.65 & 1.78 & T.m. \\
\hline $8-5,60-62$ & 72.0 & 0.00 & 5.21 & 3.62 & 0.04 & 0.43 & 0.05 & 41 & 88 & 27 & 17 & 66 & $<6$ & 2.91 & 2.02 & T-si. \\
\hline $10-2,89-91$ & 86.6 & 0.00 & 0.48 & 1.22 & 0.01 & 0.13 & 0.01 & 26 & 50 & 10 & 8 & 26 & $<6$ & 0.56 & 3.48 & D \\
\hline $11-1,100-110$ & 94.7 & 0.00 & 0.78 & 0.86 & 0.004 & 0.08 & 0.01 & 28 & 38 & 26 & 8 & 16 & $<6$ & 0.49 & 3.17 & D \\
\hline $11, \mathrm{CC}$ & 103.0 & 0.00 & 0.57 & 0.85 & 0.01 & 0.08 & 0.01 & 27 & 40 & 10 & 8 & 18 & $<6$ & 0.54 & 3.11 & D \\
\hline $12-3,90-92$ & 105.5 & 0.00 & 0.60 & 0.90 & 0.009 & 0.10 & 0.03 & 27 & 34 & 12 & 16 & 18 & $<6$ & 0.49 & 2.55 & D \\
\hline $12, \mathrm{CC}$ & 108.0 & 0.00 & 0.81 & 0.53 & 0.007 & 0.05 & 0.02 & 25 & 30 & 10 & 11 & 15 & $<6$ & 0.39 & 2.70 & D \\
\hline
\end{tabular}

Site 340

\begin{tabular}{|c|c|c|c|c|c|c|c|c|c|c|c|c|c|c|c|c|}
\hline $2-1,35-37$ & 9.9 & 7.23 & 0.45 & 3.42 & 0.04 & 0.46 & 0.05 & 32 & 84 & 42 & 22 & 84 & $<6$ & 3.35 & 2.63 & D \\
\hline $2-1,42-44$ & 10.0 & 0.00 & 0.27 & 1.60 & 0.02 & 0.13 & 0.02 & 38 & 44 & 38 & 21 & 27 & $<6$ & 1.05 & 4.80 & D \\
\hline $2-1,148-150$ & 11.1 & 10.01 & 0.45 & 3.69 & 0.07 & 0.46 & 0.06 & 27 & 82 & 44 & 22 & 84 & $<6$ & 3.33 & 2.04 & D \\
\hline $2, \mathrm{CC}$ & 19.0 & 0.00 & 0.24 & 2.36 & 0.04 & 0.30 & 0.01 & 30 & 106 & 89 & 30 & 23 & $<6$ & 1.60 & 5.07 & D \\
\hline $3-4,75-77$ & 24.6 & 1.50 & 0.15 & 1.54 & 0.02 & 0.19 & 0.01 & 37 & 59 & 45 & 22 & 28 & $<6$ & 1.13 & 5.23 & D \\
\hline $3-5,75-77$ & 26.2 & 2.50 & 0.12 & 1.85 & 0.02 & 0.19 & 0.02 & 39 & 63 & 27 & 15 & 28 & $<6$ & 1.23 & 4.85 & D \\
\hline $3, \mathrm{CC}$ & 28.5 & 2.00 & 0.24 & 2.69 & 0.02 & 0.35 & 0.01 & 61 & 101 & 44 & 19 & 40 & $<6$ & 1.26 & 4.92 & D \\
\hline $4-2,75-77$ & 31.0 & 0.00 & 0.18 & 1.91 & 0.02 & 0.13 & 0.01 & 32 & 68 & 35 & 22 & 25 & $<6$ & 1.21 & 4.20 & D \\
\hline $4, \mathrm{CC}$ & 38.0 & 0.00 & 0.18 & 1.83 & 0.02 & 0.21 & 0.01 & 31 & 68 & 32 & 19 & 28 & $<6$ & 1.12 & 4.21 & D \\
\hline $5-1,70-72$ & 38.8 & 0.00 & 0.21 & 2.13 & 0.01 & 0.22 & 0.02 & 36 & 72 & 35 & 24 & 28 & $<6$ & 1.22 & 4.07 & D \\
\hline $5, \mathrm{CC}$ & 47.5 & 2.50 & 0.21 & 1.85 & 0.02 & 0.21 & 0.01 & 36 & 71 & 32 & 24 & 25 & $<6$ & 1.06 & 4.40 & D \\
\hline $6-1,60-62$ & 48.1 & 0.00 & 0.27 & 1.06 & 0.01 & 0.08 & 0.004 & 30 & 47 & 35 & 21 & 16 & $<6$ & 0.65 & 4.25 & D \\
\hline $6-2,60-62$ & 49.9 & 0.00 & 0.24 & 2.14 & 0.01 & 0.13 & 0.004 & 30 & 48 & 35 & 22 & 18 & $<6$ & 0.62 & 3.65 & D \\
\hline $6, \mathrm{CC}$ & 67.0 & 0.00 & 0.24 & 2.10 & 0.03 & 0.29 & 0.009 & 57 & 87 & 38 & 24 & 28 & $<6$ & 1.27 & 4.12 & D \\
\hline $7-0,10-12$ & 67.1 & 0.00 & 0.27 & 2.44 & 0.02 & 0.32 & 0.02 & 43 & 81 & 40 & 22 & 33 & $<6$ & 1.44 & 4.02 & D \\
\hline $7-2,95-97$ & 59.7 & 0.00 & 0.27 & 2.27 & 0.03 & 0.24 & 0.01 & 53 & 90 & 50 & 18 & 28 & $<6$ & 1.02 & 5.10 & D \\
\hline $7-5,95-97$ & 64.4 & 0.00 & 0.12 & 1.67 & 0.02 & 0.14 & 0.009 & 43 & 72 & 38 & 18 & 24 & $<6$ & 1.02 & 3.59 & D \\
\hline $7, \mathrm{CC}$ & 66.5 & 0.00 & 0.15 & 0.95 & 0.01 & 0.05 & 0.004 & 34 & 52 & 22 & 18 & 14 & $<6$ & 0.87 & 0.87 & D \\
\hline $8-2,72-74$ & 67.2 & 0.00 & 0.15 & 1.58 & 0.01 & 0.16 & 0.009 & 37 & 51 & 34 & 15 & 24 & $<6$ & 0.91 & 3.53 & D \\
\hline $8-3,72-74$ & 70.5 & 1.75 & 0.15 & 1.76 & 0.01 & 0.16 & 0.009 & 39 & 55 & 34 & 20 & 23 & $<6$ & 1.07 & 4.16 & D \\
\hline $8, \mathrm{CC}$ & 76.0 & 2.50 & 0.24 & 2.91 & 0.02 & 0.28 & 0.01 & 57 & 141 & 44 & 20 & 35 & $<6$ & 1.43 & 4.38 & D \\
\hline $9-2,70-72$ & 78.4 & 3.00 & 0.27 & 2.16 & 0.01 & 0.14 & 0.01 & 48 & 108 & - & 26 & 28 & $<6$ & 1.09 & 4.33 & D \\
\hline $9-4,70-72$ & 81.5 & 0.00 & 0.27 & 2.55 & 0.01 & 0.22 & 0.01 & 45 & 73 & 32 & 21 & 34 & $<6$ & 1.29 & 3.81 & D \\
\hline $9-5,70-72$ & 83.1 & 0.00 & 0.33 & 2.16 & 0.01 & 0.19 & 0.01 & 45 & 71 & 32 & 17 & 26 & $<6$ & 1.13 & 3.52 & D \\
\hline $9, \mathrm{CC}$ & 85.5 & 0.00 & 0.30 & 2.79 & 0.01 & 0.24 & 0.01 & 52 & 73 & 60 & 17 & 34 & $<6$ & 1.16 & 3.59 & D-R-S \\
\hline $10-1,195-197$ & 86.4 & 0.00 & 0.24 & 2.88 & 0.01 & 0.29 & 0.009 & 52 & 80 & 44 & 19 & 33 & $<6$ & 1.31 & 3.90 & D \\
\hline $10-4,50-52$ & 90.8 & 0.00 & 0.33 & 2.80 & 0.02 & 0.29 & 0.01 & 48 & 104 & 40 & 20 & 35 & $<6$ & 1.37 & 3.78 & D \\
\hline $10-5,50-52$ & 92.4 & 0.00 & 0.27 & 2.82 & 0.01 & 0.22 & 0.01 & 53 & 89 & 26 & 13 & 34 & $<6$ & 1.50 & 3.65 & D \\
\hline $10, \mathrm{CC}$ & 95.0 & 0.00 & 0.15 & 2.15 & 0.05 & 0.22 & 0.01 & 32 & 93 & 40 & 23 & 19 & $<6$ & 1.38 & 3.36 & D \\
\hline $11-2,81-83$ & 97.4 & 0.00 & 0.30 & 2.91 & 0.01 & 0.24 & 0.01 & 60 & 100 & 54 & 22 & 38 & $<6$ & 0.99 & 2.81 & D \\
\hline $11-5,81-83$ & 102.2 & 0.00 & 0.18 & 3.40 & 0.02 & 0.26 & 0.01 & 60 & 90 & 61 & 24 & 38 & $<6$ & 1.14 & 2.87 & D \\
\hline $11-6,81-83$ & 103.8 & 1.50 & 0.24 & 2.94 & 0.01 & 0.26 & 0.01 & 62 & 101 & 50 & 24 & 34 & $<6$ & 1.08 & 2.92 & D \\
\hline $11, \mathrm{CC}$ & 104.5 & 3.00 & 0.39 & 3.24 & 0.03 & 0.24 & 0.01 & 56 & 136 & 58 & 30 & 33 & $<6$ & 1.34 & 2.84 & D-R-S \\
\hline \multicolumn{17}{|l|}{ Site 341} \\
\hline 4, CC & 38.0 & 1.75 & 0.45 & 3.08 & 0.03 & 0.40 & 0.05 & 31 & 80 & 49 & 18 & 57 & $<6$ & 1.96 & 2.85 & M-gl.m. \\
\hline $5-2,50$ & 41.0 & 0.00 & 0.96 & 3.02 & 0.02 & 0.37 & - & 31 & 86 & 50 & 18 & 64 & $<6$ & 1.89 & 3.06 & $\mathrm{R}$ \\
\hline $5, \mathrm{CC}$ & 47.5 & 0.00 & 0.66 & 3.11 & 0.02 & 0.41 & - & 33 & 86 & 53 & 21 & 64 & $<6$ & 1.89 & 2.90 & D \\
\hline $6, \mathrm{CC}$ & 57.0 & 2.75 & 0.90 & 2.95 & 0.02 & 0.32 & - & 33 & 77 & 50 & 19 & 53 & $<6$ & 1.51 & 2.78 & D \\
\hline 7, CC & 66.5 & 5.50 & 0.78 & 3.94 & 0.05 & 0.48 & - & 27 & 82 & 49 & 23 & 79 & $<6$ & 2.74 & 2.99 & M-gl.m. \\
\hline $8, \mathrm{CC}$ & 76.0 & 4.00 & 0.84 & 3.82 & 0.05 & 0.50 & - & 21 & 83 & 49 & 23 & 81 & $<6$ & 2.81 & 2.04 & M-gl.m. \\
\hline $9, \mathrm{CC}$ & 85.5 & 8.26 & 0.36 & 3.97 & 0.06 & 0.43 & - & 25 & 88 & 49 & 20 & 86 & $<6$ & 2.96 & 2.29 & M-gl.m. \\
\hline
\end{tabular}


TABLE 4 - Continued

\begin{tabular}{|c|c|c|c|c|c|c|c|c|c|c|c|c|c|c|c|c|}
\hline \multirow{2}{*}{$\begin{array}{c}\text { Sample } \\
\text { (Interval } \\
\text { in } \mathrm{cm} \text { ) }\end{array}$} & \multirow{2}{*}{$\begin{array}{l}\text { Depth } \\
(\mathrm{m})\end{array}$} & \multicolumn{6}{|c|}{ Content $(\%)$} & \multicolumn{6}{|c|}{ Content $\left(\%-10^{-4}\right)$} & \multicolumn{2}{|c|}{ Content $(\%)$} & \multirow{2}{*}{$\begin{array}{c}\text { Sedimen } \\
\text { Type }^{\mathrm{a}}\end{array}$} \\
\hline & & $\mathrm{CaCo}_{3}$ & $\mathrm{C}_{\text {org }}$ & $\mathrm{Fe}$ & $\mathrm{Mn}$ & $\mathrm{Ti}$ & $\mathrm{P}$ & $\mathrm{Cu}$ & $\mathrm{Zn}$ & $\mathrm{Ni}$ & Co & $\mathrm{Cr}$ & $\mathrm{Cd}$ & $\mathrm{K}_{2} \mathrm{O}$ & $\mathrm{Na}_{2} \mathrm{O}$ & \\
\hline \multicolumn{17}{|c|}{ Site 341 - Continued } \\
\hline $10-3,109-111$ & 90.4 & 8.50 & 0.54 & 3.89 & 0.06 & 0.43 & - & 21 & 86 & 53 & 24 & 89 & $<6$ & 2.92 & 2.18 & M-gl.m. \\
\hline $11, \mathrm{CC}$ & 99.7 & 9.00 & 0.45 & 3.97 & 0.06 & 0.38 & - & 21 & 86 & 54 & 24 & 86 & $<6$ & 3.00 & 2.15 & M-gl.m. \\
\hline $12, \mathrm{CC}$ & 105.5 & 9.51 & 0.36 & 3.99 & 0.05 & 0.47 & - & 20 & 85 & 53 & 26 & 84 & $<6$ & 2.96 & 2.17 & M-gl.m. \\
\hline $13, \mathrm{CC}$ & 114.0 & 10.76 & 0.36 & 3.92 & 0.06 & 0.40 & - & 20 & 85 & 50 & 23 & 86 & $<6$ & 2.75 & 2.22 & M-gl.m. \\
\hline $14, \mathrm{CC}$ & 123.5 & 15.76 & 0.42 & 3.76 & 0.06 & 0.43 & - & 20 & 83 & 46 & 20 & 80 & $<6$ & 2.98 & 2.02 & M-gl.m. \\
\hline $16, \mathrm{CC}$ & 171.0 & 8.00 & 0.33 & 3.72 & 0.06 & 0.40 & - & 19 & 83 & 46 & 23 & 79 & $<6$ & 3.02 & 2.14 & M-gl.m. \\
\hline $18, \mathrm{CC}$ & 209.0 & 8.76 & 0.30 & 3.81 & 0.06 & 0.47 & - & 25 & 76 & 55 & 17 & 82 & $<6$ & 3.19 & 2.22 & M-gl.m. \\
\hline $19, \mathrm{CC}$ & 228.0 & 9.00 & 0.30 & 3.92 & 0.06 & 0.44 & - & 21 & 79 & 51 & 24 & 77 & $<6$ & 3.17 & 2.29 & M-gl.m. \\
\hline $20, \mathrm{CC}$ & 247.0 & 10.51 & 0.36 & 4.42 & 0.06 & 0.54 & - & 42 & 100 & 76 & 22 & 90 & $<6$ & 2.89 & 1.93 & M-gl.m. \\
\hline $21, \mathrm{CC}$ & 266.0 & 0.25 & 0.27 & 5.08 & 0.04 & 0.54 & - & 43 & 101 & 76 & 27 & 82 & $<6$ & 3.24 & 2.14 & M-gl.m. \\
\hline $23-3,10-12$ & 304.1 & 3.00 & 0.66 & 4.88 & 0.03 & 0.61 & - & 40 & 105 & 84 & 31 & 86 & $<6$ & 3.28 & 2.08 & M-gl.m. \\
\hline $24, \mathrm{CC}$ & 323.0 & 2.50 & 0.18 & 4.83 & 0.07 & 0.53 & - & 51 & 107 & 80 & 29 & 84 & $<6$ & 3.58 & 1.86 & M-gl.m. \\
\hline $25, \mathrm{CC}$ & 342.0 & 0.25 & 1.20 & 3.96 & 0.02 & 0.56 & - & 48 & 97 & 76 & 19 & 73 & $<6$ & 2.73 & 2.00 & T-si. \\
\hline $26-2,137-139$ & 354.6 & 59.04 & 0.36 & 1.45 & 0.04 & 0.17 & - & 18 & 36 & 29 & 19 & 24 & $<6$ & 0.95 & 1.39 & $\mathrm{C}$ \\
\hline $26, \mathrm{CC}$ & 361.0 & 10.00 & 0.72 & 3.57 & 0.02 & 0.46 & - & 40 & 97 & 67 & 19 & 77 & $<6$ & 2.29 & 2.21 & T-si. \\
\hline $27-2,120-122$ & 373.4 & 3.00 & 0.75 & 3.98 & 0.02 & 0.50 & - & 35 & 99 & 71 & 19 & 77 & $<6$ & 2.73 & 1.93 & Dt. \\
\hline $27, \mathrm{CC}$ & 380.0 & 9.00 & 0.75 & 4.13 & 0.02 & 0.47 & - & 35 & 98 & 69 & 29 & 77 & $<6$ & 2.45 & 1.96 & Dt. \\
\hline $28-2,100-102$ & 392.2 & 5.75 & 0.54 & 3.93 & 0.02 & 0.53 & - & 36 & 95 & 76 & 37 & 77 & $<6$ & 2.29 & 2.11 & Dt. \\
\hline $28, \mathrm{CC}$ & 399.0 & 0.50 & 0.75 & 3.42 & 0.02 & 0.39 & - & 38 & 89 & 61 & 22 & 54 & $<6$ & 2.02 & 2.33 & Dt. \\
\hline $29-2,30-32$ & 400.9 & 3.50 & 0.75 & 3.40 & 0.02 & 0.63 & - & 35 & 92 & 72 & 22 & 67 & $<6$ & 1.84 & 2.08 & T-si. \\
\hline $29, \mathrm{CC}$ & 408.5 & 0.00 & 2.10 & 3.61 & 0.02 & 0.63 & - & 38 & 90 & 72 & 22 & 72 & $<6$ & 2.21 & 1.86 & T-si. \\
\hline $30-2,35-37$ & 410.5 & 0.75 & 1.02 & 3.89 & 0.02 & 0.59 & - & 41 & 91 & 72 & 26 & 76 & $<6$ & 2.43 & 1.98 & Dt. \\
\hline $30, \mathrm{CC}$ & 418.0 & 0.00 & 0.96 & 4.04 & 0.02 & 0.60 & - & 45 & 100 & 74 & 26 & 76 & $<6$ & 2.57 & 2.06 & T-si. \\
\hline $31-2,68-70$ & 420.3 & 0.00 & 1.20 & 4.02 & 0.02 & 0.63 & - & 50 & 100 & 74 & 26 & 67 & $<6$ & 2.50 & 1.89 & T-si. \\
\hline $31, \mathrm{CC}$ & 427.5 & 0.00 & $1.20^{\circ}$ & 3.56 & 0.02 & 0.56 & - & 44 & 92 & 72 & 21 & 66 & $<6$ & 2.27 & 1.96 & T-si. \\
\hline $32-2,50-52$ & 429.6 & 0.00 & 1.59 & 3.72 & 0.02 & 0.62 & - & 44 & 98 & 69 & 19 & 64 & $<6$ & 2.17 & 1.94 & T-si. \\
\hline $32, \mathrm{CC}$ & 437.0 & 0.50 & 1.38 & 3.67 & 0.02 & 0.56 & - & 42 & 99 & 69 & 24 & 72 & $<6$ & 2.41 & 1.92 & T-si. \\
\hline $33-2,59-61$ & 439.2 & 0.50 & 2.14 & 3.59 & 0.02 & 0.62 & - & 44 & 91 & 63 & 26 & 61 & $<6$ & 2.18 & 1.85 & T-si. \\
\hline $33, \mathrm{CC}$ & 446.5 & 0.00 & 1.77 & 3.42 & 0.03 & 0.56 & - & 53 & 95 & 64 & 26 & 59 & $<6$ & 2.17 & 2.32 & T-si. \\
\hline $34-2,59-61$ & 448.7 & 2.00 & 2.46 & 3.30 & 0.02 & 0.59 & - & 61 & 99 & 74 & 24 & 67 & $<6$ & 2.24 & 2.05 & T-si. \\
\hline $34-4,62-64$ & 451.9 & 0.50 & 2.52 & 4.01 & 0.03 & 0.49 & - & 50 & 88 & 52 & 20 & 68 & $<6$ & 1.95 & 1.93 & T-si. \\
\hline $34, \mathrm{CC}$ & 456.0 & 0.25 & 1.89 & 3.96 & 0.02 & 0.55 & - & 44 & 103 & 41 & 18 & 54 & $<6$ & 1.96 & 1.95 & T-si. \\
\hline \multicolumn{17}{|l|}{ Site 342} \\
\hline $1-1,49-51$ & 0.5 & 11.26 & 0.21 & 4.30 & 0.08 & 0.40 & - & 22 & 84 & 38 & 21 & 70 & $<6$ & 3.00 & 1.80 & T.m. \\
\hline $1-3,51-53$ & 3.5 & 18.01 & 0.24 & 3.84 & 0.11 & 0.44 & - & 28 & 67 & 37 & 18 & 87 & $<6$ & 2.19 & 2.06 & T.m. \\
\hline $1, \mathrm{CC}$ & 9.0 & 6.50 & 0.63 & 4.23 & 0.08 & 0.47 & - & 32 & 81 & 41 & 24 & 92 & $<6$ & 2.56 & 2.06 & T.m. \\
\hline $2-3,120-122$ & 42.0 & 0.75 & 0.21 & 4.97 & 0.08 & 0.52 & - & 48 & 101 & 49 & 26 & 90 & $<6$ & 2.75 & 2.20 & T.m. \\
\hline $2, \mathrm{CC}$ & 47.0 & 14.76 & 0.42 & 4.86 & 0.12 & 0.49 & - & 34 & 98 & 53 & 22 & 95 & $<6$ & 2.46 & 2.05 & T.m. \\
\hline $3-1,89-91$ & 86.0 & 0.00 & 0.66 & 6.00 & 0.03 & 0.69 & - & 44 & 103 & 79 & 33 & 95 & $<6$ & 2.63 & 2.53 & T-si. \\
\hline $3-3,119-121$ & 89.6 & - & - & 5.07 & 0.03 & 0.63 & - & 57 & 102 & 53 & 21 & 103 & $<6$ & 2.58 & 2.57 & T-si. \\
\hline $3, \mathrm{CC}$ & 94.5 & - & - & 3.63 & 0.03 & 0.46 & - & 44 & 83 & 52 & 21 & 62 & $<6$ & 1.96 & 2.85 & T-si. \\
\hline $4, \mathrm{CC}$ & 132.5 & - & - & 2.50 & 0.02 & 0.25 & - & 31 & 71 & 49 & 18 & 50 & $<6$ & 1.32 & 2.78 & D-R-S \\
\hline $5-1,100-102$ & 133.6 & - & - & 2.98 & 0.02 & 0.34 & - & 28 & 72 & 37 & 19 & 60 & $<6$ & 1.70 & 2.78 & D \\
\hline $5-3,100-102$ & 136.7 & - & - & 2.53 & 0.02 & 0.30 & - & 28 & 72 & 43 & 20 & 56 & $<6$ & 1.50 & 2.78 & D \\
\hline $5-4,100-102$ & 138.3 & - & - & 2.95 & 0.02 & 0.35 & - & 34 & 71 & 46 & 19 & 57 & $<6$ & 1.45 & 2.80 & D \\
\hline $5-5,100-102$ & 139.9 & - & - & 3.14 & 0.02 & 0.35 & - & 28 & 65 & 43 & 19 & 54 & $<6$ & 1.64 & 2.69 & D \\
\hline
\end{tabular}

${ }^{\text {a} S e d i m e n t ~ t y p e: ~ M .-g l . m . ~=~ m a r i n e-g l a c i a l ~ m u d ; ~ T-s i . ~=~ t e r r i g e n o u s ~ s i l i c e o u s ~(d i a t o m) ~}$ mud; $\mathrm{D}=$ diatom ooze; T.m. = terrigenous mud; D-R-S = diatom, radiolarian, spicule ooze; $\mathrm{R}=$ radiolarian ooze; $\mathrm{C} .=$ coccolith ooze $; \mathrm{Dt} .=$ diatomite

regime allowed a high diatom productivity (sometimes radiolarians). In all probability, the favorable regime of that time was created by: (1) different circulation systems of water masses than now and in the Pleistocene, (2) active volcanic processes, and (3) upwelling of cool water on the V $\phi$ ring Plateau.

Investigations of Recent sediments (Emelyanov, 1973; Emelyanov et al., 1975) show that under upwelling areas (i.e., southwest Africa littoral) diatom oozes from, with a rich $\mathrm{C}_{\text {org }}$ content. The $\mathrm{C}_{\text {org }}$ content in Miocene sediments is 2-5 times richer than in Recent sediments.

Late Eocene diatom oozes (Site 341) were deposited in other tectonic and hydrochemical situations. Ap- parently, the late Eocene sea was deeper than in the Miocene. This conclusion is confirmed by: (1) a very rich clay fraction $(<0.01 \mathrm{~mm})$, (2) a low content of quartz and feldspar, and (3) a very low $\mathrm{C}_{\text {org }}$ and $\mathrm{P}$ content. Similar contents are typical for pelagic diatom oozes (Emelyanov et al., 1975). The role of volcanism in the Eocene was less than in the Miocene, confirmed by low concentrations of $\mathrm{Fe}, \mathrm{Ti}, \mathrm{Ni}$, and $\mathrm{Cr}$, typical for volcanogenic sediments.

The volcanic products would have been delivered from Iceland and Jan-Mayen, as well as from closer sources. Eocene diatomites of Lim-Fiord (Denmark) contain volcanic ash, possibly from the volcanic Skagerrack region (Holtedahl, 1955). The Tertiary sub- 
TABLE 5

Comparison of Chemical Composition of Sediments from the Vorring Plateau with the Percent Sediments of the Atlantic Ocean and Baltic Sea (Russian Data)

\begin{tabular}{|c|c|c|c|c|c|c|c|c|c|c|c|c|c|c|c|}
\hline \multirow{2}{*}{$\begin{array}{c}\text { Sediment } \\
\text { Type }^{\mathrm{a}}\end{array}$} & \multirow{2}{*}{$\begin{array}{l}\text { No. of } \\
\text { Samples }\end{array}$} & \multicolumn{6}{|c|}{ Content (\%) } & \multirow{2}{*}{$\begin{array}{c}\mathrm{Cu} \\
10^{-4}\end{array}$} & \multirow{2}{*}{$\begin{array}{c}\mathrm{Zn} \\
10^{-4}\end{array}$} & \multirow{2}{*}{$\stackrel{\mathrm{Ni}}{10^{-4}}$} & \multirow{2}{*}{$\begin{array}{c}\text { Co } \\
10^{-4}\end{array}$} & \multirow{2}{*}{$\begin{array}{c}\mathrm{Cr} \\
10^{-4}\end{array}$} & \multirow{2}{*}{$\begin{array}{c}\mathrm{Cd} \\
10^{-4}\end{array}$} & \multicolumn{2}{|c|}{ Content (\%) } \\
\hline & & $\mathrm{CaCO}_{3}$ & $\mathrm{C}$ & $\mathrm{Fe}$ & $\mathrm{Mn}$ & $\mathrm{Ti}$ & $\mathrm{P}$ & & & & & & & $\mathrm{K}_{2} \mathrm{O}$ & $\mathrm{Na}_{2} \mathrm{O}$ \\
\hline & \multicolumn{15}{|c|}{ Voring Plateau } \\
\hline $\begin{array}{l}\text { M-Gl. } \\
\text { (Site 339) } \\
\text { M-GL. }\end{array}$ & 13 & 8.82 & 0.54 & 3.96 & 0.05 & 0.51 & 0.06 & 29 & 85 & 40 & 17 & 88 & $<5$ & 2.86 & 1.98 \\
\hline (Site 341) & 16 & 7.19 & 0.44 & 4.06 & 0.05 & 0.47 & - & 28 & 88 & 58 & 23 & 82 & $<6$ & 2.97 & 2.22 \\
\hline $\begin{array}{l}\text { (Site 340) } \\
\text { T-Si }\end{array}$ & 31 & 1.11 & 0.24 & 2.27 & 0.02 & 0.24 & 0.01 & 43 & 79 & 40 & 20 & 32 & $<6$ & 1.29 & 3.93 \\
\hline \multirow[t]{2}{*}{ (Site 341) } & 19 & 1.92 & 1.35 & 3.75 & 0.02 & 0.54 & - & 43 & 95 & 68 & 23 & 69 & $<6$ & 2.28 & 2.02 \\
\hline & \multicolumn{15}{|c|}{ Baltic Sea } \\
\hline T.a.-p.m. & 40 & 0.73 & 3.32 & 4.25 & 0.05 & 0.39 & 0.07 & 46 & 155 & 35 & - & 95 & $<6$ & - & - \\
\hline Moraines & 8 & 14.64 & 0.37 & 2.06 & 0.04 & 0.25 & 0.06 & - & - & - & - & - & - & - & - \\
\hline & \multicolumn{15}{|c|}{ Atlantic Ocean } \\
\hline T.S. & 60 & - & 0.56 & 2.85 & 0.05 & 0.35 & 0.07 & 66 & 83 & 38 & - & 68 & $<6$ & 1.67 & 2.16 \\
\hline T.S. & 20 & - & 0.60 & 7.00 & 0.14 & 1.12 & 0.13 & 55 & 103 & 35 & - & 145 & $<6$ & 1.56 & 3.28 \\
\hline D.O. & 8 & - & 5.77 & 1.50 & 0.02 & 0.14 & 0.48 & - & - & 72 & - & 109 & $<6$ & 1.40 & 7.54 \\
\hline
\end{tabular}

${ }^{\mathrm{a}}$ M.-Gl. = marine-glacial mud, $\mathrm{D}=$ diatom ooze, D.t. = diatomite, T.a.-p.m. = terrigenous silty-clayey mud, T.S. = terrigenous sediments $($ sand, silt, clay), V.S. = volcanic sediments, D.O. = diatom ooze $($ southwest Africa shelf $), \mathrm{T}-\mathrm{Si} .=$ terrigenous siliceous mud.

sidence of the Norwegian shelf was accompanied by volcanic activity, reflected by the presence of volcanic ash and glass in the diatom oozes of the V $\phi$ ring Plateau.

However, the role of volcanism in Tertiary sedimentation was less than at present in the Norwegian sea (Emelyanov and Kharin, 1974). Low admixture of pyroclastic material and probable enriching of seawater by $\mathrm{Si}$ and $\mathrm{P}$ are the results of the influence of volcanism. No direct influence of volcanism/hydrothermal activity on the sedimentation pattern has taken place. Authigenic minerals (Fe-sulfides, glauconite) present in the lower deposits may be a result of diagenetic alterations of the volcanic material.

\section{ACKNOWLEDGMENTS}

The individuals listed below provided the data discussed in this paper. All are members of the laboratory of Geology of the Atlantic, Atlantic Branch of the P.P. Shirshov Institute of Oceanology, Academy of Sciences of the USSR, Kaliningrad. Grain size-G.V. Zharavleva and A.I. Panichina; light and heavy minerals-N.Y. Zozovaya; chemical composition-G.S. Khanchos, T.I. Khomina, J.O. Shajdurov, Z. Ch. Jablunovskaja, and A. Ivantchenko.

\section{REFERENCES}

Eltzina, G.N., 1973. X-ray investigations of calcium carbonate of Recent sediments of the Atlantic ocean: Thesis of Report, Alecsandrovo, USSR, p. 78-79.

Emelyanov, E.M., 1973. Ooze distribution and composition on the South-Western African shelf: Inst. Oceanology USSR Trans., v. 95, p. 211-238.

Emlyanov, E.M. and Kharin, G.S., 1974. Mineral and chemical composition of the bottom sediments of of the Atlantic ocean: Investigation of the problem of rift zones of the World Ocean, v. 3, Moscow (Nauka), p. 151-186.

Emelyanov, E.M., Lisitizin, A.P., and Illyin, A.V., 1975. Types of the bottom sediments of the Atlantic ocean: Kaliningradskaya pravda, Kaliningrad, p. 1-570.

Gorbunova, Z.N., 1969. X-ray method of determination of carbonates, quartz and other minerals of sediments. Litol. Mineral Res., v. 2, p. 125-129.

Holtedahl, H., 1955. On the Norwegian Terrance, primarily outside More-Romsdal: its geomorphology and sediments. Universitet I Bergen Arbok, Neturvitenskapeling rekke, No. 14.

, 1957. Geology of Norway: v. 2, p. 1-394.

1958. Some remarks on geomorphology of continental shelves of Norway, Labrador, and southeast Alaska: J. Geol., v. 66, p. 461-471. 